\title{
New Young Stars and Brown Dwarfs in the Upper Scorpius Association*
}

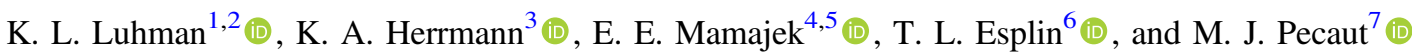 \\ ${ }^{1}$ Department of Astronomy and Astrophysics, The Pennsylvania State University, University Park, PA 16802, USA; kluhman@ astro.psu.edu \\ ${ }^{2}$ Center for Exoplanets and Habitable Worlds, The Pennsylvania State University, University Park, PA 16802, USA \\ ${ }^{3}$ The Pennsylvania State University, 1 Campus Drive, Mont Alto, PA 17237, USA \\ ${ }^{4}$ Jet Propulsion Laboratory, California Institute of Technology, 4800 Oak Grove Drive, Pasadena, CA 91109, USA \\ ${ }^{5}$ Department of Physics \& Astronomy, University of Rochester, 500 Wilson Boulevard, Rochester, NY 14627, USA \\ ${ }^{6}$ Steward Observatory, University of Arizona, 933 North Cherry Avenue, Tucson, AZ 85721, USA \\ ${ }^{7}$ Department of Physics, Rockhurst University, 1100 Rockhurst Road, Kansas City, MO 64110-2508, USA \\ Received 2018 March 21; revised 2018 May 3; accepted 2018 June 7; published 2018 July 27
}

\begin{abstract}
To improve the census of the Upper Sco association ( $\sim 11 \mathrm{Myr}, \sim 145 \mathrm{pc})$, we have identified candidate members using parallaxes, proper motions, and color-magnitude diagrams from several wide-field imaging surveys and have obtained optical and infrared spectra of several hundred candidates to measure their spectral types and assess their membership. We also have performed spectroscopy on a smaller sample of previously known or suspected members to refine their spectral types and evidence of membership. We have classified 530 targets as members of Upper Sco, 377 of which lack previous spectroscopy. Our new compilation of all known members of the association contains 1631 objects. Although the census of Upper Sco has expanded significantly over the last decade, there remain hundreds of candidates that lack spectroscopy. The precise parallaxes and proper motions from the second data release of Gaia should extend down to substellar masses in Upper Sco, which will greatly facilitate the identification of the undiscovered members.
\end{abstract}

Key words: brown dwarfs - stars: formation - stars: low-mass - stars: luminosity function, mass function - stars: pre-main sequence

Supporting material: data behind figures, machine-readable tables

\section{Introduction}

The Upper Scorpius subgroup in the Scorpius-Centaurus OB association is located at a distance of $\sim 145 \mathrm{pc}$, has an age of $\sim 11 \mathrm{Myr}$, and contains $>1000$ members (Preibisch \& Mamajek 2008; Pecaut et al. 2012), making it the largest stellar population at $\lesssim 10 \mathrm{Myr}$ within $300 \mathrm{pc}$. As such, Upper Sco offers some of the best available constraints on statistical properties of young stellar populations, such as their initial mass function (IMF), stellar multiplicity, planet occurrence rate, and disk fraction. The association is old enough that its natal cloud has dispersed, so its members have only modest extinction $\left(A_{V} \lesssim 3\right)$ and can be observed at optical wavelengths. However, because the members are distributed across a large area of sky $\left(\sim 100 \mathrm{deg}^{2}\right)$, it has been challenging to obtain a complete census of the association. Surveys for members of Upper Sco have utilized wide-field imaging at X-ray, ultraviolet, optical, and infrared (IR) wavelengths (Walter et al. 1994; Martín 1998; Martín et al. 1998, 2004, 2010; Preibisch et al. 1998, 2001, 2002; de Zeeuw et al. 1999; Ardila et al. 2000; Hoogerwerf 2000; Lodieu et al. 2006, 2007, 2008, 2011a, 2011b, 2013a, 2013b, 2018; Slesnick et al. 2006, 2008; Torres et al. 2006; Findeisen \& Hillenbrand 2010; Dawson et al. 2011, 2014; Rizzuto et al. 2011, 2015; Lodieu 2013; Pecaut \& Mamajek 2016; Peña Ramírez et al. 2016; Cook et al. 2017; Wilkinson et al. 2018). Members uncovered through that work have served as targets in surveys to find substellar companions through direct imaging (Kraus et al. 2005, 2008;

\footnotetext{
* Based on observations made with the Two Micron All Sky Survey, the Deep Near-Infrared Survey of the Southern Sky, the Wide-field Infrared Survey Explorer, the United Kingdom Infrared Telescope Infrared Deep Sky Survey, the Visible and Infrared Survey Telescope for Astronomy Hemisphere Survey, Pan-STARRS1, the Gaia mission, the NASA Infrared Telescope Facility, Cerro Tololo Inter-American Observatory, and Gemini Observatory.
}

Kraus \& Hillenbrand 2007, 2009; Béjar et al. 2008; Lafrenière et al. 2010, 2011, 2014; Biller et al. 2011; Ireland et al. 2011; Aller et al. 2013; Hinkley et al. 2015; Uyama et al. 2017), detect and characterize circumstellar disks via IR and millimeter observations (Rieke et al. 2005; Chen et al. 2005, 2011; Carpenter et al. 2006, 2008, 2009, 2014; Padgett et al. 2006; Scholz et al. 2007; Dahm \& Carpenter 2009; Riaz et al. 2009, 2012; Luhman \& Mamajek 2012; Mathews et al. 2012, 2013; Rizzuto et al. 2012, 2015; Dawson et al. 2013; Jang-Condell et al. 2015; Barenfeld et al. 2016, 2017), and measure photometric variability from a variety of phenomena (e.g., eclipsing binaries, transiting planets) with Kepler's K2 mission (Alonso et al. 2015; Kraus et al. 2015; Lodieu et al. 2015; Ripepi et al. 2015; Scholz et al. 2015; Ansdell et al. 2016; David et al. 2016a, 2016b, 2017; Mann et al. 2016; Scaringi et al. 2016; Cody et al. 2017; Rizzuto et al. 2017; Stauffer et al. 2017, 2018; Cody \& Hillenbrand 2018; Hedges et al. 2018; Rebull et al. 2018) as well as the Super Wide Angle Search for Planets (Mellon et al. 2017) and the Kilodegree Extremely Little Telescope (Ansdell et al. 2018).

Although extensive work has been done in identifying the members of Upper Sco, the current census likely has substantial incompleteness, particularly among low-mass stars and brown dwarfs. To improve the completeness of the census in Upper Sco, we are performing a search for new members using photometry and astrometry from optical and IR widefield surveys, which include the Two Micron All Sky Survey (2MASS; Skrutskie et al. 2006), the Deep Near-Infrared Survey of the Southern Sky (DENIS; Epchtein et al. 1999), the United Kingdom Infrared Telescope Infrared Deep Sky Survey (UKIDSS; Lawrence et al. 2007), the Wide-field Infrared Survey Explorer (WISE; Wright et al. 2010), Pan-STARRS1 (PS1; Kaiser et al. 2002, 2010), the Gaia mission (Perryman 


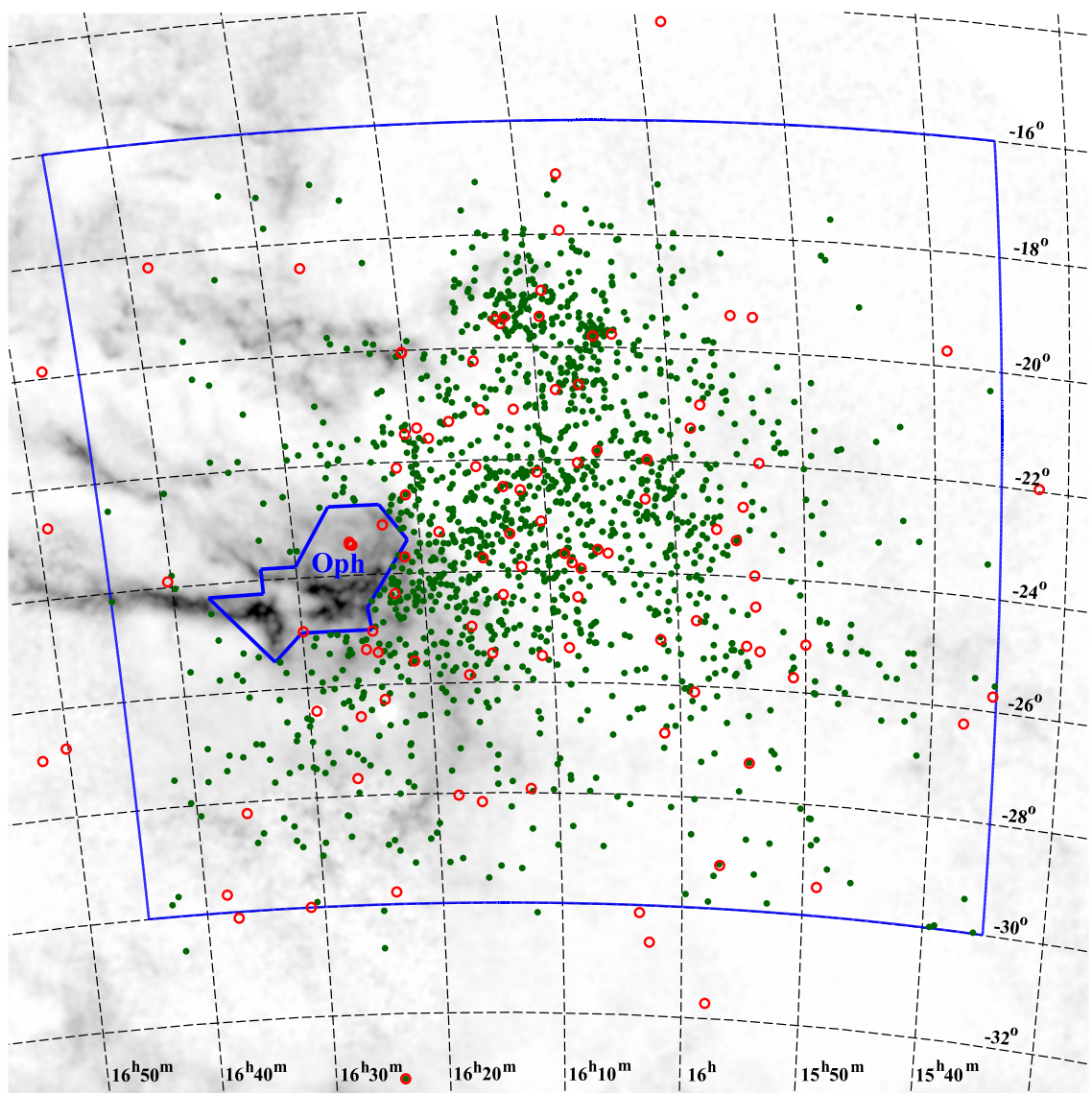

Figure 1. Spatial distribution of stars identified as probable members of Upper Sco by de Zeeuw et al. (1999) using Hipparcos astrometry (open circles), excluding 12 stars that are nonmembers based on spectral classifications or Gaia parallaxes. We also include all other stars that we have adopted as members of the association (filled circles, Table 1). In our survey for new members of Upper Sco, we have considered the area from $\alpha=15^{\mathrm{h}} 35^{\mathrm{m}}$ to $16^{\mathrm{h}} 45^{\mathrm{m}}$ and $\delta=-30$ to $-16^{\mathrm{o}}($ rectangle) and outside of the Ophiuchus region (small polygon). The Ophiuchus dark clouds and the diffuse clouds across portions of Upper Sco are displayed with a map of extinction (gray scale, Dobashi et al. 2005).

et al. 2001; de Bruijne 2012), and the Visible and Infrared Survey Telescope for Astronomy (VISTA) Hemisphere Survey (VHS; McMahon et al. 2013). In this paper, we present the new members found to date through our survey and a compilation of all known members of Upper Sco.

\section{Known Members of Upper Sco}

During a study of circumstellar disks in Upper Sco, Luhman \& Mamajek (2012) compiled a list of known members of the association. That list contained 863 sources, 181 of which comprised an initial sample of new members found through the survey that we are presenting in this work. Many additional members have been identified by subsequent surveys of Upper Sco. We have revised and expanded the compilation of members from Luhman \& Mamajek (2012) to account for those surveys and new membership constraints, as summarized in this section.

To compile known members of Upper Sco, we began by considering the 120 stars (mostly B through $\mathrm{F}$ types) that were identified as probable members by de Zeeuw et al. (1999) based on proper motions and parallaxes from the Hipparcos mission. We have excluded eight candidates that have been spectroscopically classified as field stars (Houk 1982; Houk \& SmithMoore 1988; Chen et al. 2011; Pecaut et al. 2012), which consist of HIP 75916, HIP 78271, HIP 79247, HIP 79258, HIP 79596, HIP 82140, HIP 83456, and HIP 83542. The first data release of the Gaia mission (Gaia Collaboration et al. 2016a, 2016b) has provided new parallax measurements for 76 of the 112 remaining candidates. Several stars exhibit discrepant Gaia parallaxes relative to the bulk of the sample. Among those stars, we assigned membership to those that are known or suspected to be binaries (HIP 78233, HIP 78581, HIP 80896), as the presence of a close companion can lead to erroneous astrometry. Another candidate with a discrepant parallax, HIP 82218, is treated as a member because of the abundance of evidence of its membership (Chen et al. 2011). We reject HIP 78963, HIP 79860, HIP 79987, and HIP 80586 based on their parallaxes (and radial velocity for HIP 80586, Nordström et al. 2004).

The positions of the 108 unrejected stars from de Zeeuw et al. (1999) are plotted on the map in Figure 1. Based on the distribution of those stars, we selected $15^{\mathrm{h}} 35^{\mathrm{m}}$ and $16^{\mathrm{h}} 45^{\mathrm{m}}$ in right ascension $(\alpha)$ and $-30^{\circ}$ to $-16^{\circ}$ in decl. $(\delta)$ as the boundaries for our survey, which encompass 97 of the 108 probable members. In Figure 1, we have marked our adopted boundary between Upper Sco and the stellar population associated with the Ophiuchus dark clouds, which was defined by Esplin et al. (2018). Five of the stars from de Zeeuw et al. (1999) appear within that boundary for Ophiuchus, and hence 
are excluded from our membership list for Upper Sco. The 103 remaining stars outside of Ophiuchus are included in the list.

We have compiled additional members of Upper Sco from the previous surveys cited in Section 1, a survey for diskbearing members by Esplin et al. (2018), a proper motion survey of Ophiuchus that extends into Upper Sco (T. Esplin 2018, in preparation), and the survey that we are presenting. We have also considered young stars that have been found in the vicinity of Upper Sco through studies that were not explicitly searching for members of the association (e.g., Herbig \& Kameswara Rao 1972; Gizis 2002; Allers et al. 2006; Shkolnik et al. 2009; Kirkpatrick et al. 2010; Romero et al. 2012; Best et al. 2017). We have adopted stars as members if they are located between the boundary of Ophiuchus and the outer boundary of our survey field (Figure 1), have measured spectral types, exhibit evidence of youth, and have parallaxes or proper motions that are similar to those of the adopted members from de Zeeuw et al. (1999) when available. We have included a few additional stars outside of our survey field that have been adopted as members in multiple previous studies (see Figure 1). Diagnostics of youth consist of $\mathrm{Li}$ I absorption, gravity-sensitive absorption features (e.g., $\mathrm{Na}$, $\mathrm{FeH}, \mathrm{H}_{2} \mathrm{O}$ ), and mid-IR excess emission that is indicative of a circumstellar disk. The first two diagnostics are not applicable to B and A stars, and many members of young populations lack disks, so we do not require evidence of youth for members at those types. The membership classification of a star can change as new data become available (e.g., Muzerolle et al. 2003; Bouy \& Martín 2009), so some stars previously identified as members do not appear in our compilation and some stars previously classified as field stars are now adopted as members. We have attempted to consider all available membership constraints for each candidate that we have examined.

In Table 1, we list the stars that we have adopted as known members of Upper Sco. We have included the available measurements of spectral types, the types that we adopt, and our estimates of extinction (Section 4.2). This compilation contains 1631 objects. Spectra have been obtained for 185, 31, and 530 members by Esplin et al. (2018), T. Esplin (2018, in preparation), and this work, respectively, 166, 31, and 377 of which have not been observed with spectroscopy previously.

\section{Identification of Candidate Members}

\subsection{Sources of Data}

Because the members of Upper Sco are distributed across a large area of sky, we have used wide-field imaging surveys to search for members of the association. The surveys have provided photometry in several optical and IR bands: $J H K_{s}$ from the 2MASS Point Source Catalog, $i$ from the third data release of DENIS, ZYJHK from the science verification release and data release 10 of UKIDSS, $W 1$ and $W 2^{8}$ from the AllWISE Source Catalog, YJHK from the fifth data release of VISTA VHS, $G$ from the first data release of Gaia, and rizy $y_{P 1}$ (Tonry et al. 2012) from the first data release of the $3 \pi$ survey from PS1 (Chambers et al. 2016; Flewelling et al. 2016). Additional bands are available from some of these catalogs, but they are redundant with data adopted from other surveys. Some of the catalogs provide multiple methods of measuring

\footnotetext{
WISE obtained images in bands at $3.5,4.5,12$, and $22 \mu \mathrm{m}$, which are denoted as $W 1, W 2, W 3$, and $W 4$, respectively.
}

Table 1

Members of Upper Sco

\begin{tabular}{ll}
\hline \hline Column Label & Description \\
\hline 2MASS & 2MASS Point Source Catalog source name \\
WISEA & AllWISE Source Catalog source name \\
UGCS & UKIDSS Galactic Clusters Survey source name \\
Names & Other source names \\
RAdeg & R.A. (J2000) \\
DEdeg & Decl. (J2000) \\
SpType & Spectral type \\
r_SpType & Spectral type reference \\
Adopt & Adopted spectral type \\
Ak & Extinction in $K_{s}$ \\
f_Ak & Method of extinction estimation \\
\hline
\end{tabular}

Notes.

a The following names are from the WISE All-Sky Source Catalog: J160027.15223850.5, J160414.16-212915.5, J161320.78-175752.3, J161317.38-292220.0, J161837.22-240522.8, J162210.14-240905.4, J162230.38-241119.2, J162620.15223312.8 .

b Based on coordinates from Data Release 10 of the UKIDSS Galactic Clusters Survey for stars with $K_{s}>10$ from 2MASS.

c (1) Houk \& Smith-Moore (1988), (2) this work, (3) Kunkel (1999), (4) Preibisch et al. (1998), (5) Pecaut \& Mamajek (2016), (6) Torres et al. (2006), (7) Esplin et al. (2018), (8) Rizzuto et al. (2015), (9) Dawson et al. (2014), (10) measured in this work with the most recently published spectrum, (11) Lodieu et al. (2006), (12) Lodieu et al. (2008), (13) Bonnefoy et al. (2014), (14) Ruiz et al. (1987), (15) Best et al. (2017), (16) Houk (1982), (17) Vieira et al. (2003), (18) Peña Ramírez et al. (2016), (19) Pecaut et al. (2012), (20) Corbally (1984), (21) Shkolnik et al. (2009), (22) Aller et al. (2013), (23) Ardila et al. (2000), (24) Walter et al. (1994), (25) Martín et al. (2010), (26) Kraus \& Hillenbrand (2009), (27) Martín et al. (2004), (28) Slesnick et al. (2008), (29) Preibisch et al. (2002), (30) Mora et al. (2001), (31) Reid et al. (2008), (32) Kirkpatrick et al. (2010), (33) Allers \& Liu (2013), (34) Faherty et al. (2016), (35) Slesnick et al. (2006), (36) Riaz et al. (2006), (37) Lodieu et al. (2018), (38) Kraus et al. (2015), (39) Cody et al. (2017), (40) Lafrenière et al. (2011), (41) Lachapelle et al. (2015), (42) Preibisch et al. (2001), (43) Ansdell et al. (2016), (44) Béjar et al. (2008), (45) Herczeg et al. (2009), (46) Kraus \& Hillenbrand (2007), (47) Lodieu et al. (2011a), (48) Biller et al. (2011), (49) Lafrenière et al. (2008), (50) Luhman et al. (2017), (51) Mann et al. (2016), (52) David et al. (2016b), (53) Stauffer et al. (2017), (54) Stauffer et al. (2018), (55) Cohen \& Kuhi (1979), (56) Prato et al. (2003), (57) Eisner et al. (2005), (58) Gizis (2002), (59) Martín et al. (1998), (60) Prato (2007), (61) Cowley et al. (1969), (62) Lodieu et al. (2015), (63) David et al. (2016a), (64) Luhman (2005), (65) Martín (1998), (66) T. Esplin (2018, in preparation); (67) Gray et al. (2006); (68) Lutz \& Lutz (1977), (69) Cieza et al. (2010), (70) Bowler et al. (2011), (71) Bowler et al. (2014), (72) Romero et al. (2012), (73) Jayawardhana \& Ivanov (2006), (74) Close et al. (2007), (75) Luhman et al. (2007), (76) Merín et al. (2010), (77) McClure et al. (2010), (78) David et al. (2017), (79) Brandner \& Zinnecker (1997), (80) Bouvier \& Appenzeller (1992), (81) Bowler et al. (2017), (82) Wilking et al. (2005), (83) Erickson et al. (2011), (84) Cieza et al. (2007).

${ }^{\mathrm{d}}$ Extinction estimated from a near-IR spectrum or the indicated color assuming the intrinsic spectrum from Luhman et al. (2017) or the intrinsic color from Kenyon \& Hartmann (1995) and Luhman et al. (2010) for the spectral type in question.

(This table is available in its entirety in machine-readable form.)

photometry for a given band. We adopted the $1^{\prime \prime}$ radius aperture magnitudes from UKIDSS and VHS and the pointspread-function magnitudes from the stacked images in PS1. To avoid saturated measurements, we excluded PS1 data brighter than $15 \mathrm{mag}$, UKIDSS data at $Z<11.4, Y<11.3$, $J<10.5, H<10.2$, and $K<9.7$, and VHS data at 2MASS magnitudes of $J<13, H<13$, and $K_{s}<12.5$. We also omitted $Y$ data from VHS for $J<13$. When photometry was available at two epochs in $K$ from UKIDSS, we adopted the 


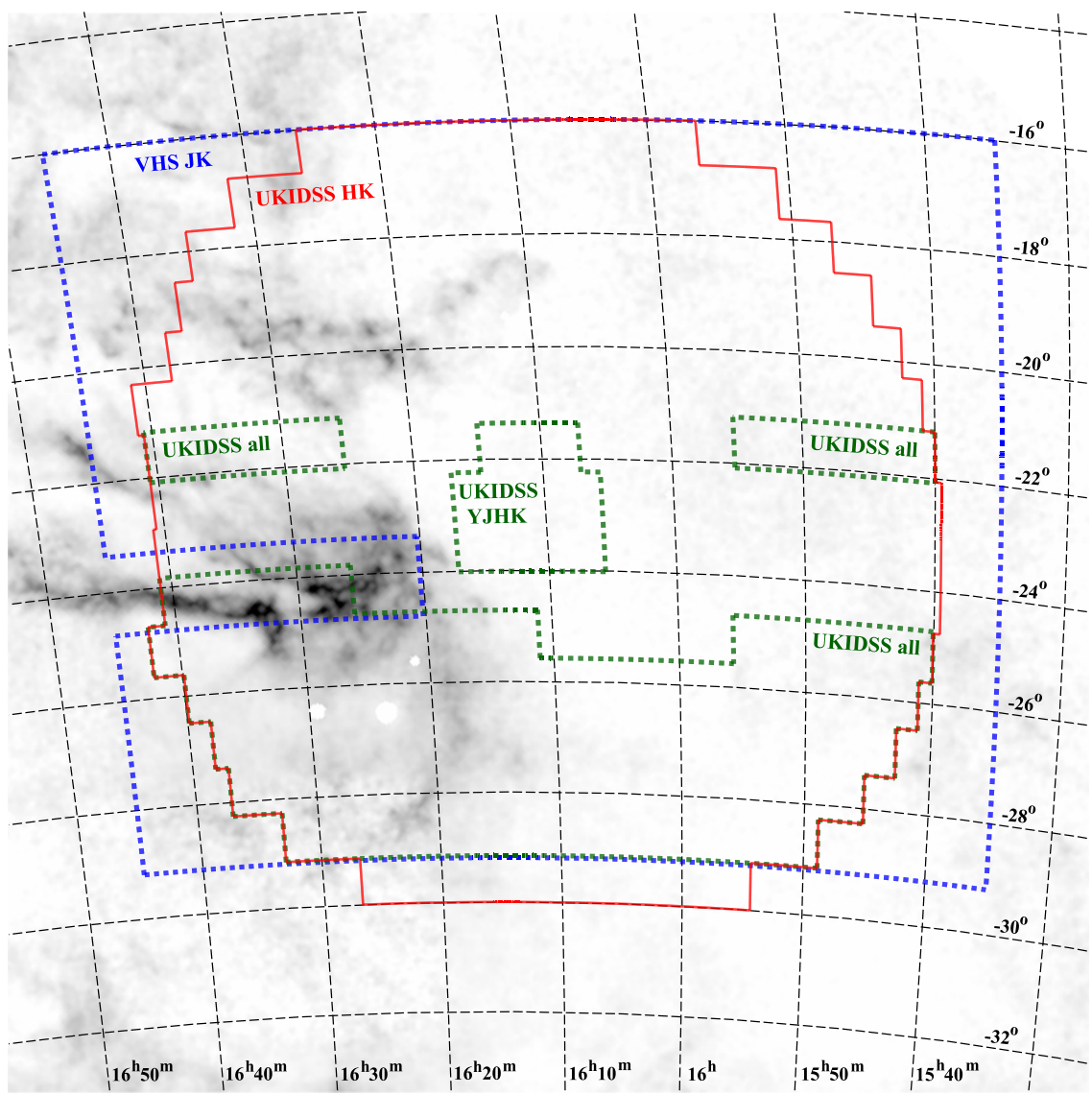

Figure 2. Areas in our survey field of Upper Sco that have been imaged by UKIDSS and VISTA VHS. The clouds in Ophiuchus and Upper Sco are displayed with a map of extinction (gray scale, Dobashi et al. 2005).

average of those measurements. For some of the strips in the DENIS catalog, all of the measurements exhibited unusually large photometric errors. We did not use data from those strips. In addition to the above photometry, we have utilized proper motions and parallaxes from Gaia DR1 and proper motions from UKIDSS, the fifth release of the U.S. Naval Observatory CCD Astrograph Catalog (UCAC5; Zacharias et al. 2017), and the Gaia-PS1-Sloan Digital Sky Survey (GPS1) Proper Motion Catalog (Tian et al. 2017). ${ }^{9}$

We combined the above catalogs by first identifying all matching sources. To merge the photometry from different surveys, we treated the following filters as the same: $J H K_{s}$ (2MASS) $/ J H K$ (UKIDSS) $/ J H K$ (VHS) and $Y$ (UKIDSS) / $Y$ (VHS). For $J H K_{s}$, we adopted 2MASS measurements if the errors were $\leqslant 0.06$. When the 2MASS errors were larger than that threshold, we adopted the measurements with the smallest errors from among 2MASS, UKIDSS, and VHS. For $Y$, we used the measurements with the smallest errors from UKIDSS and VHS. The bands $Z$ (UKIDSS) $/ z_{P 1}$ and $Y$ (UKIDSS) $/ y_{P 1}$ are sufficiently different that they are used separately in our analysis.

The images from 2MASS, WISE, Gaia, and PS1 cover all or nearly all of our survey field in Upper Sco. After excluding the strips with large photometric errors, the remaining DENIS data covered $\sim 85 \%$ of the survey field. The boundaries of the areas imaged by UKIDSS and VHS are indicated on the map in Figure 2. Those areas were not fully imaged by those surveys;

\footnotetext{
9 In addition to the databases listed in its name, GPS1 also employed astrometry from 2MASS
}

small patches within them lack data. The coverage of UKIDSS in Upper Sco was also presented by Lodieu (2013). Most of the available VHS data were obtained in $J$ and $K$. Only a very small portion of Upper Sco has been imaged by VHS in $Z$ and $H$, which is not shown in Figure 2. Imaging has been performed in small areas of Upper Sco that is deeper than the wide-field surveys cited above (Lodieu et al. 2013a, 2018; Peña Ramírez et al. 2016). Those data are not utilized in our survey, as only the raw images are publicly available.

\subsection{Proper Motions and Parallaxes}

We have used the surveys that we have compiled to identify candidate members of Upper Sco via parallaxes, proper motions, and color-magnitude diagrams (CMDs). We describe our selection criteria for the parallaxes and proper motions in this section. Our analysis of the CMDs is discussed in the next section.

Measurements of parallaxes are available from Gaia DR1 for 169 of the brightest stars in our compilation of known members of Upper Sco $(G \sim 5-11.5)$. The median value of these parallax data corresponds to $146 \mathrm{pc}$, which agrees with previous distance estimates for the association (Preibisch \& Mamajek 2008). Most of the parallaxes have $1 \sigma$ errors that overlap with a distance range of $125-165 \mathrm{pc}$, so we have adopted this criterion when identifying candidate members among other stars that have parallaxes from Gaia DR1.

To select candidates based on proper motions, we have utilized the motions measured by UKIDSS, GPS1, and UCAC5. The GPS1 catalog contains multiple options for 
proper motion that differ in their fits or combinations of data. Two of those options, the robust fit and cross-validated fit, use all available data from Gaia DR1, PS1, 2MASS, and Sloan (the latter does not cover Upper Sco). We compared the measurements from those two fits for the known members of Upper Sco. The formal errors from the robust fit are smaller than those from the cross-validated fit, but the two sets of data exhibit similar dispersions, which would suggest that they have similar errors. We have adopted the motions from the cross-validated fit, as they contain fewer outliers relative to the average motion of the members. We also have computed one set of proper motions from a combination of 2MASS and Gaia positions and a second set of motions from a combination of 2MASS and PS1 positions. The PS1 data were based on coadditions of images from multiple epochs. In our analysis, we have excluded proper motion measurements with errors

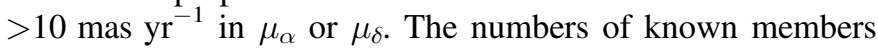
with proper motion measurements are 169 for Gaia $(G \sim 5-11.5), 929$ for GPS1 $(G \sim 12.5-18.5), 823$ for UCAC5 $\quad(G \sim 4.5-15.5), \quad 1297$ for $2 \mathrm{MASS} /$ Gaia $(G \sim 5-21), 895$ for 2 MASS $/$ PS1 $(G \gtrsim 13)$, and 382 for UKIDSS $(G \gtrsim 16)$. 2MASS/PS1 and UKIDSS provide motions for a few hundred members that are below the detection limit of Gaia $(G \gtrsim 21)$.

As with the parallaxes, we have used known members of Upper Sco to design our proper motion criteria for selecting candidate members. We began by estimating the intrinsic spread in motions within Upper Sco using the most accurate measurements that are available, which are those from Gaia. Those data are plotted in a diagram of $\mu_{\delta}$ versus $\mu_{\alpha}$ in Figure 3 . They exhibit a dispersion $\left(\sigma \sim 3\right.$ mas $\left.\mathrm{yr}^{-1}\right)$ that is significantly larger than that expected from measurement errors $\left(\sim 0.3 \mathrm{mas} \mathrm{yr}^{-1}\right)$ or projection effects that occur across the area covered by Upper Sco $\left(0.5-1 \mathrm{mas} \mathrm{yr}^{-1}\right)$. Thus, it appears that the scatter is dominated by the dispersion in kinematics and distances among the members (see also Wright \& Mamajek 2018), and thus reflects the intrinsic spread in the proper motions of the association. Most of the known members with

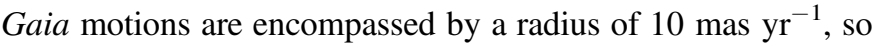
we have adopted that threshold for our selection of candidates. For each of the six sources of proper motions that we have utilized, we identified stars that have $1 \sigma$ errors that overlap with

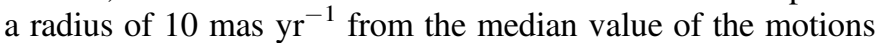
of the known members. We also identified stars that are beyond the threshold by more than $2 \sigma$. Measurements at $1-2 \sigma$ are

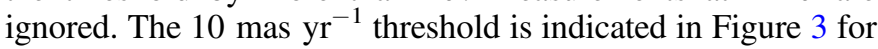
each source of proper motions. We classified each star as either a candidate member or a probable field star based on the first data set in which it was flagged as within $1 \sigma$ or beyond $2 \sigma$ of Upper Sco from among Gaia, GPS1, UCAC5, 2MASS/Gaia, 2MASS/PS1, and UKIDSS. Given this approach, a star with one proper motion measurement that indicates nonmembership can be considered a candidate if another measurement from a more preferred source supports membership. A small number of stars in our compilation of known members would be rejected by this criterion (i.e., they appear as outliers in Figure 3), but they have been retained as members because they are known or suspected binaries, which can lead to erroneous proper motion measurements, or they have proper motions from other sources that are consistent with membership.

We note that Cook et al. (2017) measured a dispersion of

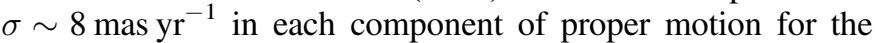

known members using data from GPS1, UCAC5, and Altmann et al. (2017). They concluded that their measurement was dominated by the kinematic spread of the population. However, as discussed earlier, the more accurate Gaia motions show a significantly smaller dispersion $\left(\sigma \sim 3\right.$ mas $\left.\mathrm{yr}^{-1}\right)$, indicating that the dispersion from Cook et al. (2017) primarily reflected measurement errors.

\subsection{Color-Magnitude Diagrams}

We have constructed CMDs for our survey field in Upper Sco using the photometry described in Section 3.1. For these diagrams, we have selected $K_{s}$ (or $K$ ) as the vertical axis and colors that contain this band because it offers the greatest sensitivity to low-mass members of Upper Sco among the available bands. For colors, that band is paired with $G, i$ from DENIS, $Z$ and $Y$ from UKIDSS, $i_{P 1}, z_{P 1}, y_{P 1}$, and $H$. We also have included a diagram with $W 1-W 2$. Although the natal molecular cloud of Upper Sco has dispersed, non-negligible dust extinction $\left(A_{V} \sim 1-3\right)$ is present across portions of the association, particularly near the Ophiuchus clouds (Figure 1), which increases contamination of background stars in regions of CMDs that are inhabited by association members. To reduce this contamination, we have estimated extinctions for individual stars in the manner described by Esplin \& Luhman (2017; see also Luhman et al. 2003) and have dereddened their positions in most of the CMDs accordingly. The one exception is the diagram of $K_{s}$ versus $H-K_{s}$, where we use the observed colors. In this analysis, we have adopted $A_{H} / A_{K}=1.55$ (Indebetouw et al. 2005) and the reddening relations from Schlafly et al. (2016), Xue et al. (2016, references therein), and Danielski et al. (2018), which produce $A_{G} / A_{K}=6.8, A_{i(P 1)} / A_{K}=6.51, A_{z(P 1)} / A_{K}=$ 5.12, $A_{y(P 1)} / A_{K}=4.17, A_{J} / A_{K}=2.62, A_{W 1} / A_{K}=0.6$, and $A_{W 2} / A_{K}=0.48$. For $i$ from DENIS and $Z$ and $Y$ from UKIDSS, we have estimated relations of $A_{i} / A_{K}=6.1, A_{Z} / A_{K}=5$, and $A_{Y} / A_{K}=3.8$ based on the values of $E\left(i-K_{s}\right) / E(J-H)$, $E\left(Z-K_{s}\right) / E(J-H)$, and $E\left(Y-K_{s}\right) / E(J-H)$ for reddened stars in our survey field.

In Figure 4, we present the extinction-corrected CMDs for the known members of Upper Sco, including those found in this work. The CMD with the observed data in $H$ and $K_{s}$ is shown in Figure 5. In each diagram, we have defined a boundary that follows the lower edge of the sequence of known members, which we have used for selecting candidate members for spectroscopy. A few members appear below some of those boundaries; most of them exhibit mid-IR excess emission that indicates the presence of circumstellar material, so it is plausible that their low positions in the CMDs are due to scattered light dominating their observed fluxes.

A source is considered a candidate member if it is selected with at least one of the CMDs in Figure 4, is not rejected by any of those diagrams, is a candidate in $K_{s}$ versus $H-K_{s}$ in Figure 5, and is not a probable field star based on the analysis of parallaxes and proper motions in the previous section. We inspected the resulting candidates in images from the employed surveys and rejected those that appear to be galaxies. In the next section, we describe spectroscopy of some of the candidates that we have identified in this manner, which is used to measure their spectral types and assess their membership. Because we have added proper motions and CMDs to our selection process as they have become available, some of the targets in our spectroscopic sample that were observed early in our survey would now be rejected 


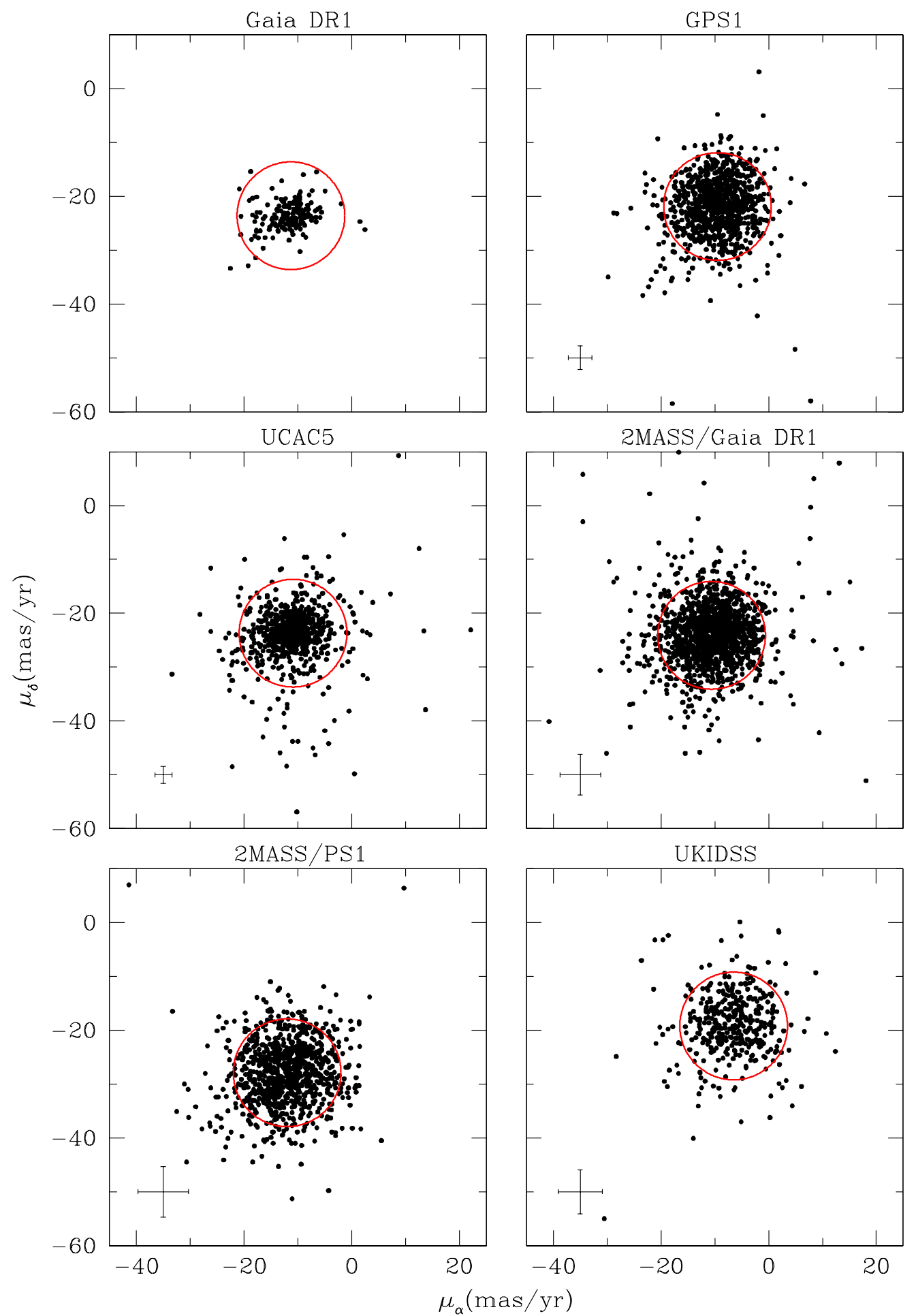

Figure 3. Proper motions for known members of Upper Sco from Gaia DR1, GPS1, UCAC5, 2MASS/Gaia DR1, 2MASS/PS1, and UKIDSS. Most of the outliers are known or suspected binaries, which can lead to erroneous proper motion measurements, or have proper motions from other sources that are consistent with membership. For a given set of data, the large circle represents the threshold that we have used for identifying new candidate members (see Section 3.2). The typical errors are indicated in the corner of each diagram except for Gaia DR1, for which most errors are similar to the sizes of the points.

by our latest criteria. In Table 2, we present the candidates that lack spectroscopy or that have insufficient information from the available spectra to determine membership. This sample contains 1196 objects. We indicate in Table 2 the selection criteria that were satisfied by each candidate. As noted in the previous section, it is possible for a candidate to be rejected by a given proper motion measurement as long as a more preferred source of proper motions favors membership. To illustrate the distribution of magnitudes of these candidates, we have plotted them in a diagram of $K_{s}$ versus $H-K_{s}$ in Figure 5. We have also included diagrams of $J-H$ versus $H-K_{s}$ for the known members and the candidates. The candidates exhibit similar $J-H$ and $H-K_{s}$ colors as the known members. 

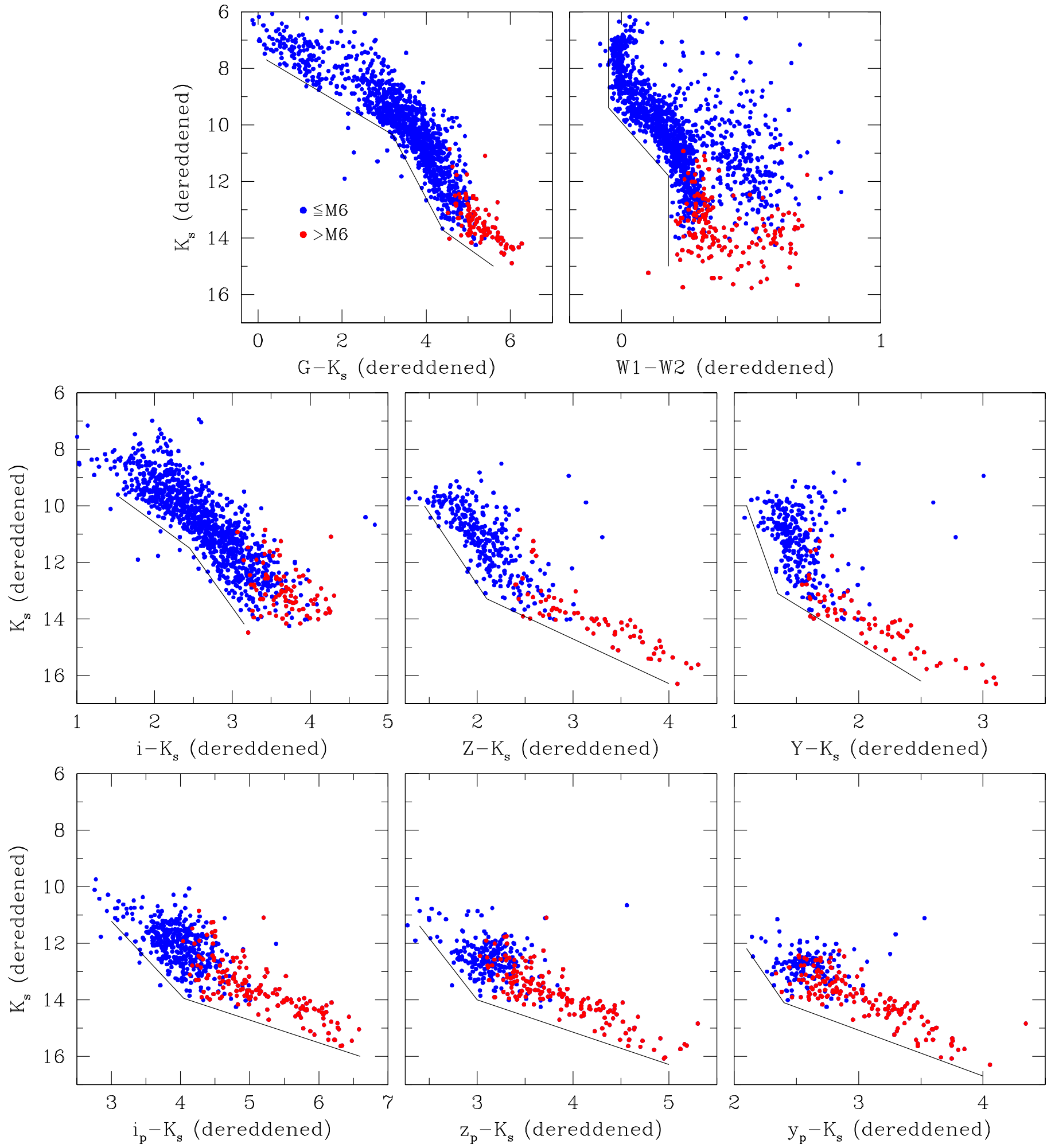

Figure 4. Extinction-corrected CMDs for the known members of Upper Sco. These data are from 2MASS, DENIS, UKIDSS, PS1, VISTA VHS, WISE, and Gaia (Section 3.1). We have selected candidate members from the other stars detected in these surveys based on positions above the solid boundaries.

\section{Spectroscopy of Candidates}

\subsection{Observations}

We have obtained optical and near-IR spectra of candidate members of Upper Sco from the previous section to measure their spectral types and assess their membership. We also have observed a sample of stars that were previously known or suspected to be members to improve their spectral classifications and evidence of membership. These data were taken between 2009 and 2017 at the $1.5 \mathrm{~m}$ SMARTS and $4 \mathrm{~m}$ Blanco telescopes at the Cerro Tololo Inter-American Observatory (CTIO), the NASA Infrared Telescope Facility (IRTF), the Southern Astrophysical Research Telescope (SOAR), and the Gemini North and South telescopes. We preferred optical 
known members
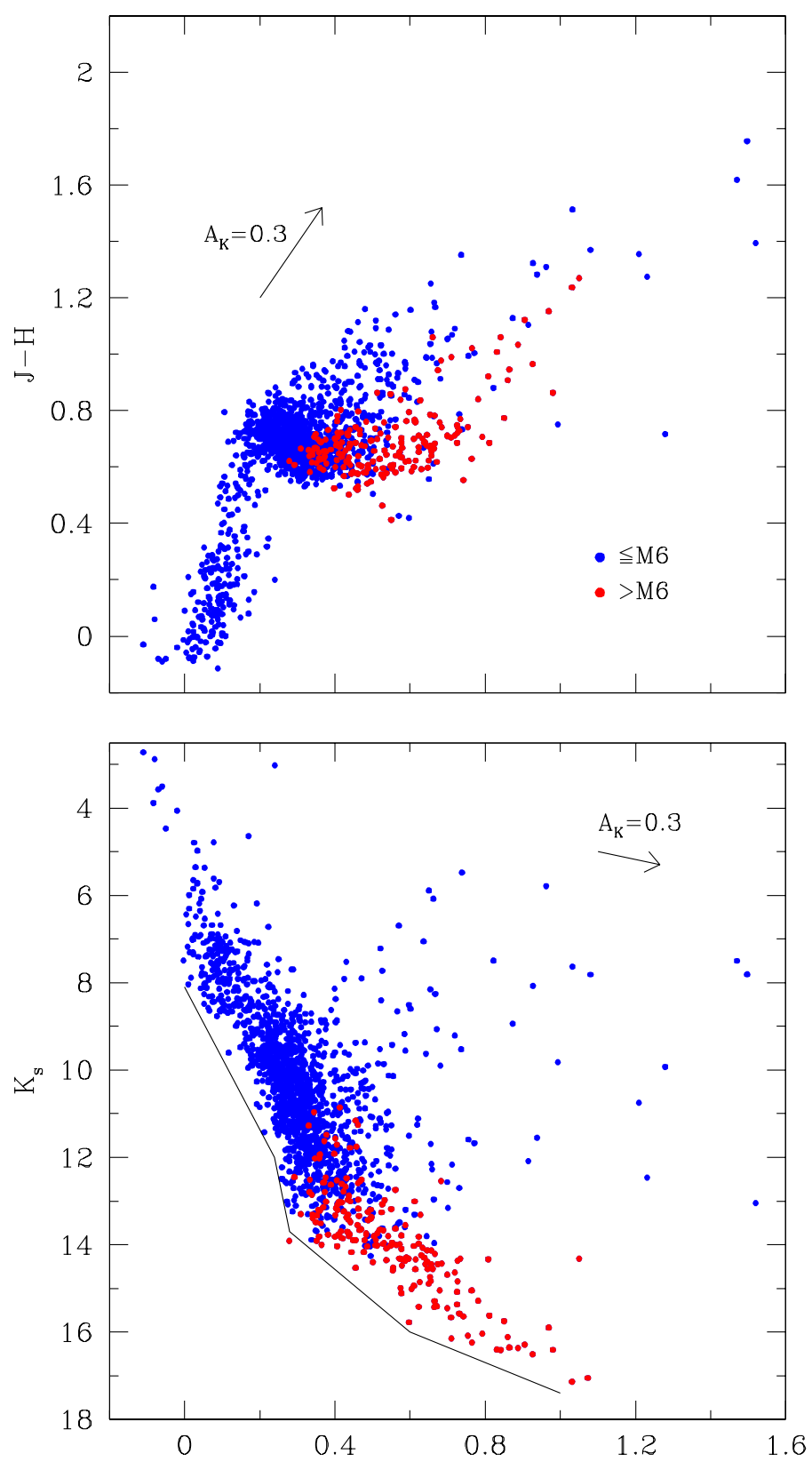

candidate members
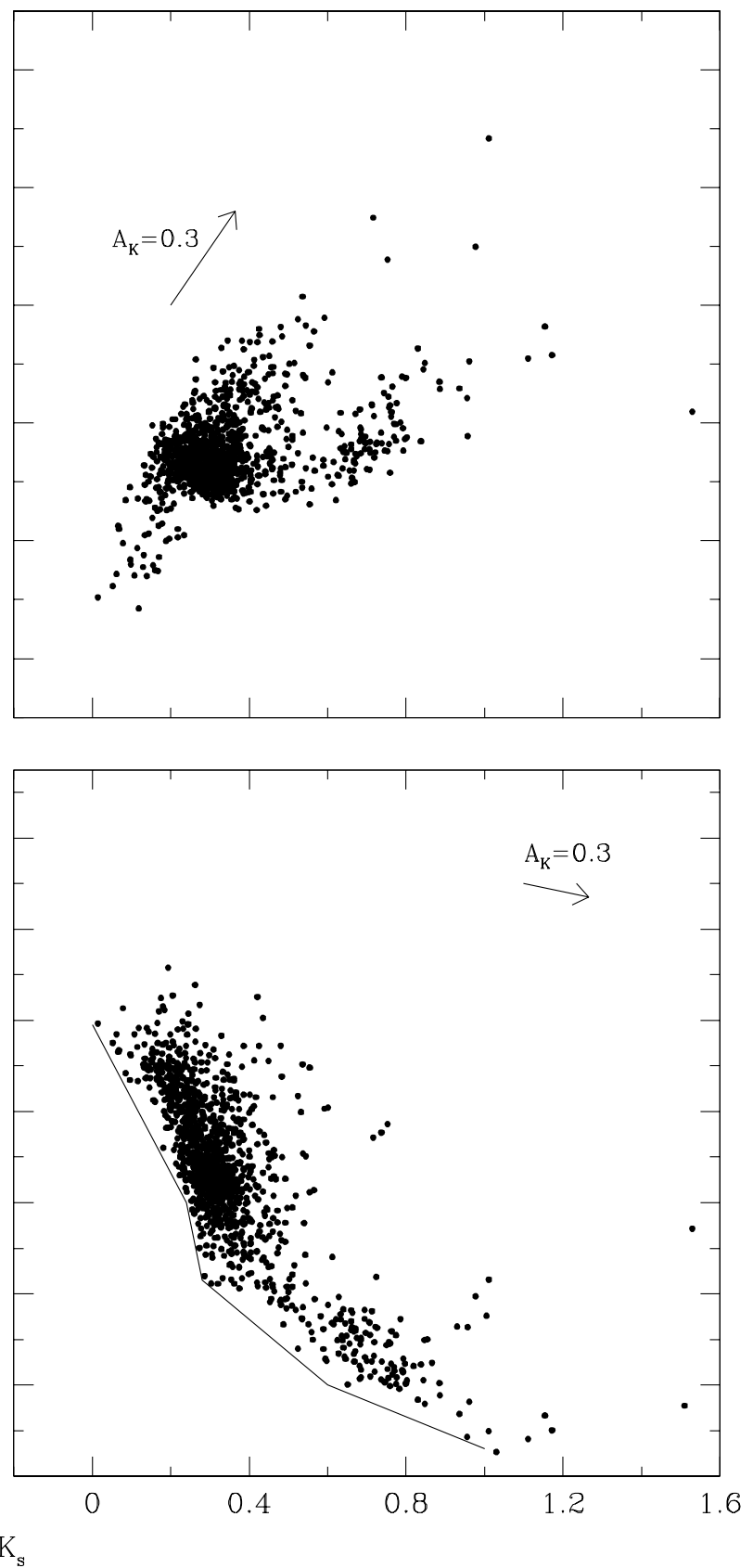

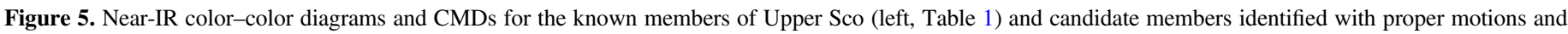

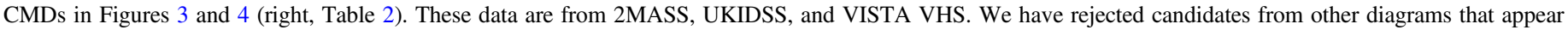
below the solid boundary in $K_{s}$ vs. $H-K_{s}$.

spectroscopy for targets that were sufficiently bright since the spectral types of young stars are defined at optical wavelengths and Li absorption at $6707 \AA$ is a valuable diagnostic of youth. For targets that were too faint for Li measurements or accurate optical classifications, we employed near-IR spectroscopy instead. The instrument configurations are summarized in Table 3. Spectroscopy was performed on 768 objects. The date and instrument for each target are indicated in Table 4.

During the several years of our spectroscopic survey, our selection criteria were updated to incorporate new photometry and astrometry as they became available. In addition, to utilize as many fibers as possible during the multi-object observations with Hydra, we included targets that failed some selection criteria. For these reasons, some of the spectroscopic targets do not fully satisfy our latest set of criteria.

The spectra from the RC spectrograph were reduced in the manner described by Pecaut \& Mamajek (2016). The SpeX data were reduced with the Spextool package (Cushing et al. 2004) and corrected for telluric absorption (Vacca et al. 2003). The spectra from the other instruments were reduced using routines within IRAF. Examples of the reduced optical and IR spectra of young objects are shown in Figures 6 and 7, respectively. All of the reduced spectra from our survey are provided in electronic files that accompany those figures. We have included the SpeX data for 2MASS J16183317$2517504 \mathrm{~A}$ and B from Luhman (2005). The slopes for the 
Table 2

Candidate Members of Upper Sco

\begin{tabular}{ll}
\hline \hline Column Label & Description \\
\hline 2MASS & 2MASS Point Source Catalog source name \\
WISEA & AllWISE Source Catalog source name \\
UGCS & UKIDSS Galactic Clusters Survey source name \\
RAdeg & R.A. (J2000) \\
DEdeg & Decl. (J2000) \\
SpType & Spectral type \\
r_SpType & Spectral type reference \\
Jmag & $J$ magnitude \\
e_Jmag & Error in Jmag \\
Hmag & $H$ magnitude \\
e_Hmag & Error in Hmag \\
Ksmag & $K_{s}$ magnitude \\
e_Ksmag & Error in Ksmag \\
JHKref & $J H K_{s}$ reference \\
selection & Selection criteria satisfied by candidate \\
\hline
\end{tabular}

Notes.

${ }^{\text {a }}$ Based on coordinates from Data Release 10 of the UKIDSS Galactic Clusters Survey for stars with $K_{s}>10$ from 2MASS.

b (1) Esplin et al. (2018), (2) this work, (3) Houk \& Smith-Moore (1988), (4) Slesnick et al. (2008), (5) Houk (1982), (6) Rizzuto et al. (2015), (7) Cieza et al. (2007).

${ }^{\mathrm{c}} 2=2$ MASS Point Source Catalog; $\mathrm{u}=$ UKIDSS Data Release 10; $\mathrm{v}=$ VISTA VHS Data Release 5.

${ }^{\mathrm{d}} \mathrm{G} / \mathrm{W} / \mathrm{i} / \mathrm{Y} / \mathrm{Z} / \mathrm{ip} / \mathrm{zp} / \mathrm{yp}=\mathrm{CMDs}$ in Figure 4 (all candidates also satisfy the $H-K_{s}$ diagram in Figure 5); pi = parallax from Gaia DR1; gaia/gps/ucac/ $2 \mathrm{~m}$-gaia/2m-ps/ukidss $=$ proper motions in Figure 3.

(This table is available in its entirety in machine-readable form.)

fiber spectra from Hydra are not reliable, particularly near the ends of the spectra. All of the slit observations were performed with the slit rotated to the parallactic angle, so their spectral slopes should be accurate. Many of the Goodman spectra contain significant fringing at redder wavelengths. The spectra from the RC spectrograph were taken with two settings that produced spectra that are separated by a gap in wavelength. The two spectra for a given target are contained within a single file and have arbitrary normalizations relative to each other.

\subsection{Classifications of New Spectra}

To classify the optical and IR spectra that we have collected in Upper Sco, we have applied the same methods that we have utilized in our previous surveys of nearby star-forming regions. We distinguished between young stars (which are likely to be members of the association) and field dwarfs and giants using using $\mathrm{Li}$ absorption and gravity-sensitive features (e.g., Na, $\mathrm{H}_{2} \mathrm{O}$ ), as available. When present, IR excess emission and strong hydrogen emission indicate the presence of a circumstellar accretion disk, providing additional evidence of youth. For targets that are classified as dwarfs or giants, we have measured spectral types through comparison to the optical and IR spectra of standards (Keenan \& McNeil 1989; Kirkpatrick et al. 1991, 1997; Henry et al. 1994; Cushing et al. 2005; Rayner et al. 2009). We also classified the optical and IR spectra of young stars at $<\mathrm{M} 5$ and $<\mathrm{M} 0$, respectively, in the same way. The optical spectra of young stars at M5-M9.5 were classified with averages of the dwarf and giant standards (Luhman et al. 1997, 1998; Luhman 1999). We adopted the optical spectra of the youngest field $\mathrm{L}$ dwarfs from Cruz et al. $(2007,2009)$ and Kirkpatrick et al. $(2006,2010)$ as standards for $>$ M9.5 (spectral type suffixes of $\delta$ or $\gamma$, corresponding to $\sim 10-100$ Myr), although none of our optical spectra of young objects exhibited types in that range. To classify the IR spectra of young stars at $\geqslant \mathrm{M} 0$, we applied the standard spectra described by Luhman et al. (2017), which were measured for optically classified members of nearby star-forming regions (1-10 Myr) at M0-M9.5, the youngest optically classified dwarfs in the field at L0, L2, and L4 (Kirkpatrick et al. 2006, 2010; Cruz et al. 2007, 2009, 2018), and two members of the TW Hya association that have been previously assigned types of L7 (Kellogg et al. 2015; Schneider et al. 2016). By adopting IR standards of this kind for young objects, our classifications should produce IR types that are on the same system as the optical types. When comparing a target to a standard at a given spectral type, reddening was artificially applied to the standard to match the spectral slope of the target.

We now describe how we used the Li absorption at $6707 \AA$ and the Na doublet near $8190 \AA$ to assess the youth of our targets that were observed with optical spectroscopy. Our measurements of the equivalent widths of these lines are included in Table 4. Those data are reported only for dwarfs and young stars, and the Na data are provided only for M types since they are not useful as a gravity diagnostic at earlier types. For some of the stars with optical spectra, the signal-to-noise ratios were too low for useful measurements. The typical strengths of $\mathrm{Li}$ and $\mathrm{Na}$ depend on both age and spectral type, so we plot them as a function of spectral type in Figure 8. We also show upper envelopes for Li data in IC 2602 (30 Myr) and the Pleiades (125 Myr) from Neuhäuser et al. (1997) and measurements of $\mathrm{Na}$ for a sample of standard field dwarfs from our previous surveys and Filippazzo et al. (2016). Li data are available from Rizzuto et al. (2015) for a few stars that lack useful $\mathrm{Li}$ constraints in our spectra, so we have adopted them. In Figure 8, most of the $\mathrm{Li}$ detections are stronger than expected for the two comparison clusters at 30 and $125 \mathrm{Myr}$, indicating ages that are consistent with that of Upper Sco (11 Myr, Pecaut et al. 2012; Feiden 2016). The remaining detections fall along the upper envelopes for those clusters and could be consistent with Upper Sco membership given the uncertainties. Therefore, we take all of the Li detections in Figure 8 as evidence of membership.

In Figure 8, the standard field dwarfs exhibit stronger $\mathrm{Na}$ than most of the young, Li-bearing stars in our spectroscopic sample, which is a reflection of the dependence of this feature on surface gravity. The two groups overlap at M0-M3 and diverge at later types. The $\mathrm{M}$ stars in our sample with useful upper limits on their $\mathrm{Li}$ absorption $(<0.2 \AA)$ have stronger $\mathrm{Na}$ on average than the Li-bearing stars, which is consistent with the older ages implied by their $\mathrm{Li}$ constraints $(\gtrsim 20 \mathrm{Myr}$, Chabrier \& Baraffe 1997; D'Antona \& Mazzitelli 1997; Siess et al. 2000). To use $\mathrm{Na}$ for identifying targets that are likely to be young enough to be members of Upper Sco, we have defined a boundary in Figure 8 that is just below nearly all of the stars at $\geqslant \mathrm{M} 3.5$ that lack Li strong absorption. Measurements of $\mathrm{Na}$ below this boundary are taken as evidence of membership unless Li absorption is absent. Stars that appear above the boundary and that lack useful constraints on Li can be considered possible members if they have other evidence of membership, such as IR excess emission, or have noticeable reddening, which is not expected for foreground field dwarfs.

In Table 4, we list the spectral types and membership classifications for the 768 objects in our spectroscopic sample. 


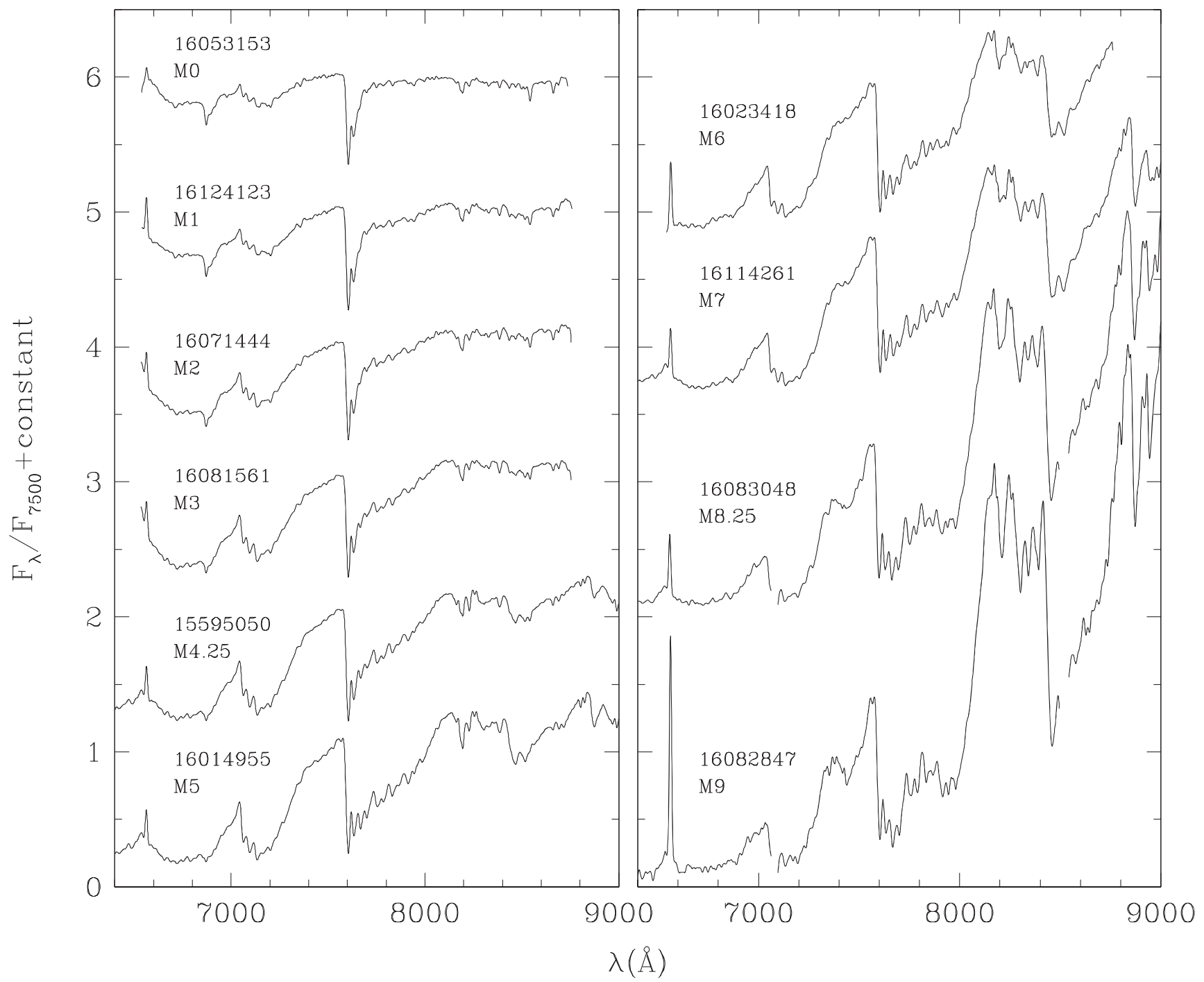

Figure 6. Examples of optical spectra of members of the Upper Sco association. These data are displayed at a resolution of $13 \AA$. The data used to create this figure are available.

Table 3

Observing Log

\begin{tabular}{|c|c|c|c|}
\hline Telescope/Instrument ${ }^{\mathrm{a}}$ & Disperser/Aperture & Wavelengths/Resolution & Targets \\
\hline CTIO 4 m/Hydra & KPGLF $/ 2^{\prime \prime}$ fiber & $0.65-0.87 \mu \mathrm{m} / 4 \AA$ & 289 \\
\hline CTIO 4 m/COSMOS & red $\mathrm{VPH} / 0.9$ or 1 ". 2 slit & $0.55-0.95 \mu \mathrm{m} / 3$ or $4 \AA$ & 105 \\
\hline CTIO $1.5 \mathrm{~m} / \mathrm{RC}$ Spec & 600 and $8311 \mathrm{~mm}^{-1} / 2^{\prime \prime}$ slit & $0.37-0.69 \mu \mathrm{m} / 3-4 \AA$ & 9 \\
\hline SOAR/Goodman & $4001 \mathrm{~mm}^{-1} / 0^{\prime \prime} 45 \mathrm{slit}$ & $0.54-0.94 \mu \mathrm{m} / 3 \AA$ & 74 \\
\hline Gemini South/GMOS & $\mathrm{R} 400 / 0$ "'75 slit & $0.57-0.99 \mu \mathrm{m} / 6 \AA$ & 15 \\
\hline Gemini North/GNIRS & $31.71 \mathrm{~mm}^{-1} / 1^{\prime \prime}$ slit & $0.9-2.5 \mu \mathrm{m} / R=600$ & 8 \\
\hline IRTF/SpeX & prism $/ 0$ ". 8 slit & $0.8-2.5 \mu \mathrm{m} / R=150$ & 287 \\
\hline
\end{tabular}

Note.

a The Gemini Multi-Object Spectrograph (GMOS), the Gemini Near-Infrared Spectrograph (GNIRS), and SpeX are described by Hook et al. (2004), Elias et al. (2006), and Rayner et al. (2003), respectively. The Cerro Tololo Ohio State Multi-Object Spectrograph (COSMOS) is based on an instrument described by Martini et al. (2011).

Stars with membership flags of either "Y" or "Y?" are included in our compilation of adopted members of Upper Sco in Table 1. Among the 530 spectroscopic targets that are adopted as members, 377 have not been previously observed with spectroscopy. Stars that have undetermined membership (marked as "?") and that satisfy our latest set of selection criteria from the CMDs and proper motions are included in the sample of candidate members in Table 2. For the stars 
Table 4

Spectroscopic Data for Previously Known and Candidate Members of Upper Sco

\begin{tabular}{|c|c|c|c|c|c|c|}
\hline Source Name ${ }^{a}$ & Spectral Type ${ }^{b}$ & $\begin{array}{c}W_{\lambda}(\mathrm{Li}) \\
(\AA)\end{array}$ & $\begin{array}{c}W_{\lambda}(\mathrm{Na}) \\
(\AA)\end{array}$ & Instrument & Date & Member? \\
\hline 2MASS J15350863-2532397 & M5.25 & $\ldots$ & $\ldots$ & SpeX & 2012 Apr 23 & $\mathrm{Y}$ \\
\hline 2MASS J15355111-2021008 & M7.5 & $\ldots$ & $\ldots$ & SpeX & 2012 Apr 22 & $\mathrm{Y}$ \\
\hline 2MASS J15374943-1920571 & G3 & 0.27 & $\cdots$ & RC Spec & 2011 May $13 /$ Jun 16 & $\mathrm{~N}$ ? \\
\hline 2MASS J15411302-2308161 & M5.5 & $\cdots$ & $\cdots$ & SpeX & 2012 Apr 24 & $\mathrm{Y}$ \\
\hline 2MASS J15413401-2507482 & M9 & $\cdots$ & $\cdots$ & SpeX & 2013 Jun 18 & Y \\
\hline
\end{tabular}

Notes.

${ }^{\text {a }}$ Source names are from the 2MASS Point Source Catalog when available. Otherwise, they are from the UKIDSS Galactic Clusters Survey.

${ }^{\mathrm{b}}$ Uncertainties are 0.25 and 0.5 subclass for optical and IR spectral types, respectively, unless indicated otherwise.

(This table is available in its entirety in machine-readable form.)

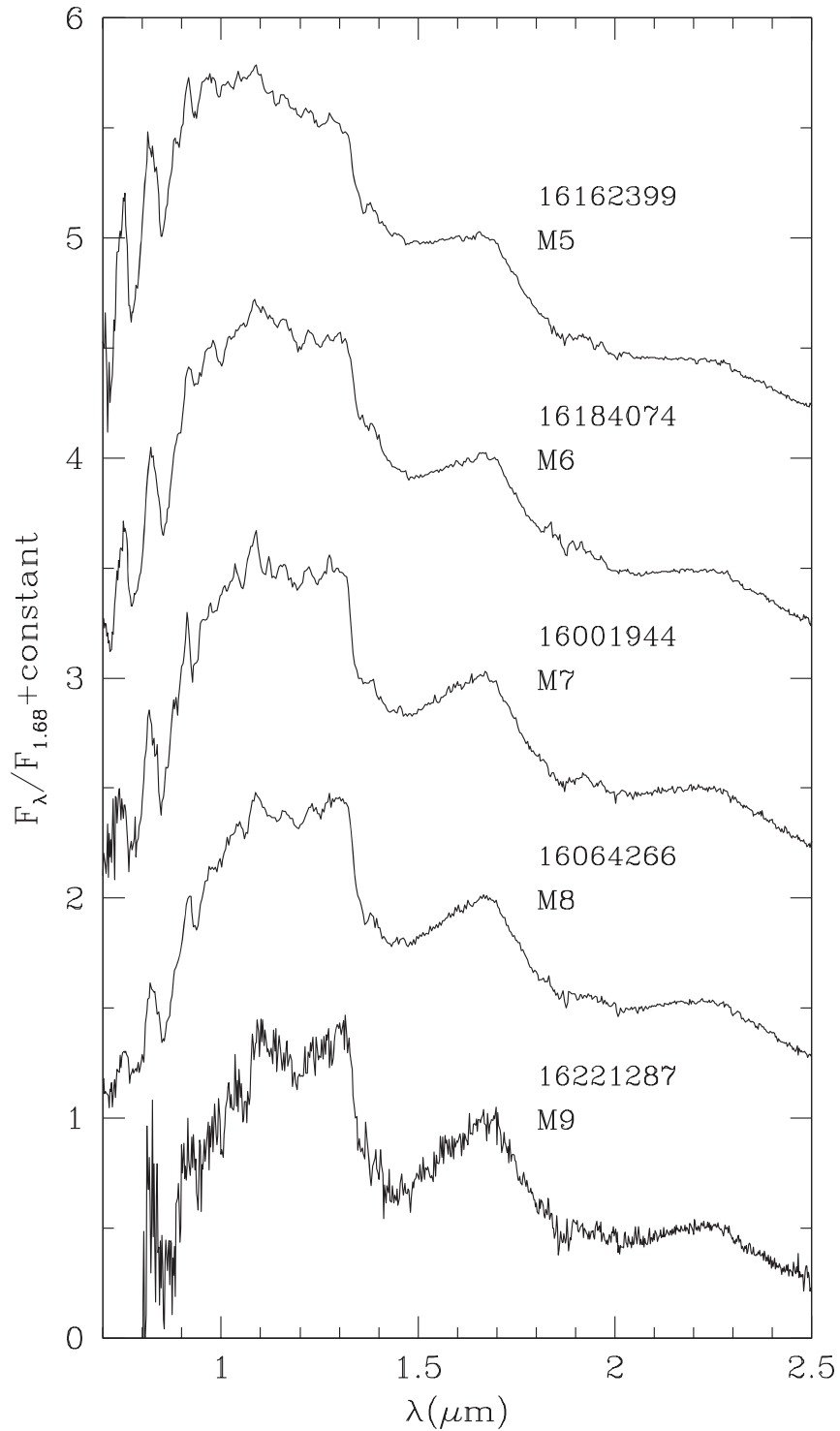

Figure 7. Examples of near-IR spectra of M-type members of the Upper Sco association. They have been dereddened to match the slopes of the young standards from Luhman et al. (2017). The data used to create this figure are available.

classified as members that have IR spectra, we have estimated extinctions by comparing the observed spectral slopes to the slopes of young standards from Luhman et al. (2017). For members that lack IR spectra, we have derived extinctions from color excesses in $J-H$ relative to the intrinsic photospheric values from Kenyon \& Hartmann (1995) and Luhman et al. (2010). The companion HD 144218 has $K_{s}$ but not $J$, so $V-K_{s}$ was used instead. The resulting extinction estimates in $K_{s}$ are included in Table 1 . We have not attempted to estimate an extinction for Antares, which is a supergiant, or the companion 2MASS J16054012-2317155E, which lacks the necessary data.

\subsection{Classifications of Previous Spectra}

In addition to classifying the objects in our spectroscopic sample, we have measured new spectral types from IR spectra that have been obtained by previous studies for late-type ( $\geqslant$ M5) members of Upper Sco (Luhman 2005; Lodieu et al. 2008, 2018; Allers \& Liu 2013; Bowler et al. 2014, 2017; Dawson et al. 2014; Lachapelle et al. 2015; Peña Ramírez et al. 2016; Best et al. 2017). When deriving spectral types, some of these studies have assumed that the targets are not reddened by interstellar dust (or have the same extinction as a more massive companion, as in the case of Bowler et al. 2017). However, according to our extinction estimates, 85\%, 10\%, and $4 \%$ of the known members have $A_{K}=0-0.15,0.15-0.3$, and $0.3-0.5$, respectively $\left(A_{K} \approx 0.1 A_{V}\right)$. Thus, reddening was a free parameter in our classification of the previous spectra, as was done for our spectroscopic sample. In general, allowing for the possibility of reddening results in a larger range of spectral types that can match a spectrum. This is particularly true for $\mathrm{L}$ types since it is primarily the spectral slope that varies with type rather than the depths of the absorption bands in lowresolution data (see Figure 17 in Luhman et al. 2017). Our new classifications are included in the compilation of spectral types for the known members in Table 1.

Lodieu et al. (2008) did not reduce the two orders at $0.9-1.15 \mu \mathrm{m}$ in their GNIRS data, so we have performed a new reduction to include them. In Figure 9, we show an example of one of the newly reduced spectra, which is compared to young standards from Luhman et al. (2017) for our derived spectral type and the type from Lodieu et al. (2008). All of these newly reduced spectra are provided in the electronic file that accompanies Figure 9.

In Figure 10, we show additional examples of previously reported spectra that we have classified. The spectra are for UGCS J155150.21-213457.2 and UGCS J160413.03 -224103.2 (Peña Ramírez et al. 2016; Lodieu et al. 2018). 


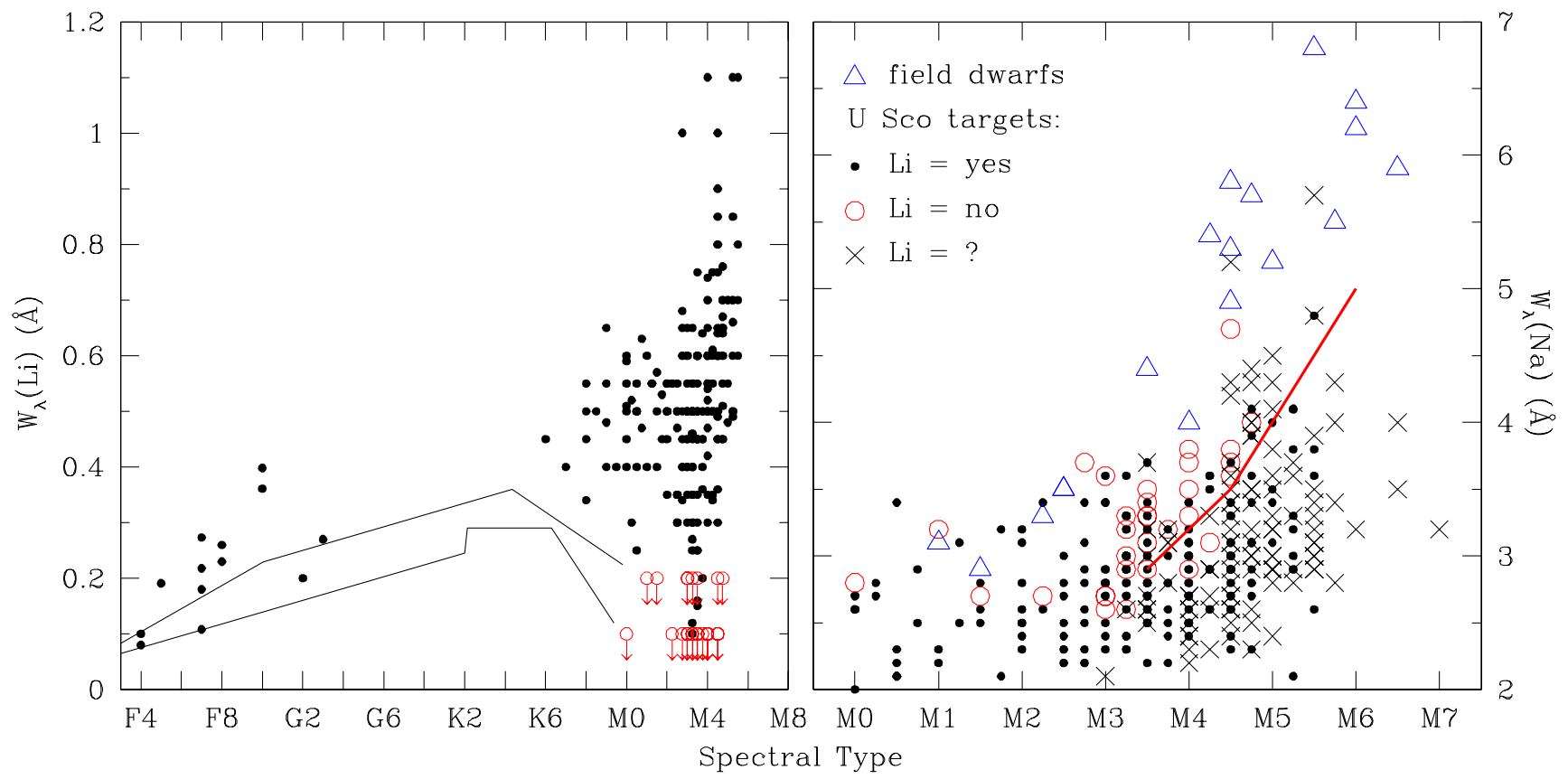

Figure 8. Equivalent widths of $\mathrm{Li}$ and $\mathrm{Na}$ vs. spectral type for previously known and candidate members of Upper Sco for which we have obtained optical spectra (Table 4). The left diagram contains detections of $\mathrm{Li}$ (filled circles) and the useful upper limits $(<0.2 \AA)$ that are available for non-detections (open circles). In the right diagram, we show Na measurements for those two samples and the stars that lack useful constraints on Li (crosses). For comparison, we include the upper envelopes for Li data in IC 2602 (30 Myr) and the Pleiades (125 Myr) (upper and lower solid lines, Neuhäuser et al. 1997) in the left diagram and Na measurements for a sample of field dwarfs in the right diagram. Most of these Li detections are consistent with the youth expected for members of Upper Sco. The Na strengths for dwarfs and young, Li-bearing stars diverge noticeably at $\gtrsim \mathrm{M} 3.5$. We take Na measurements below the red curve as evidence of youth.

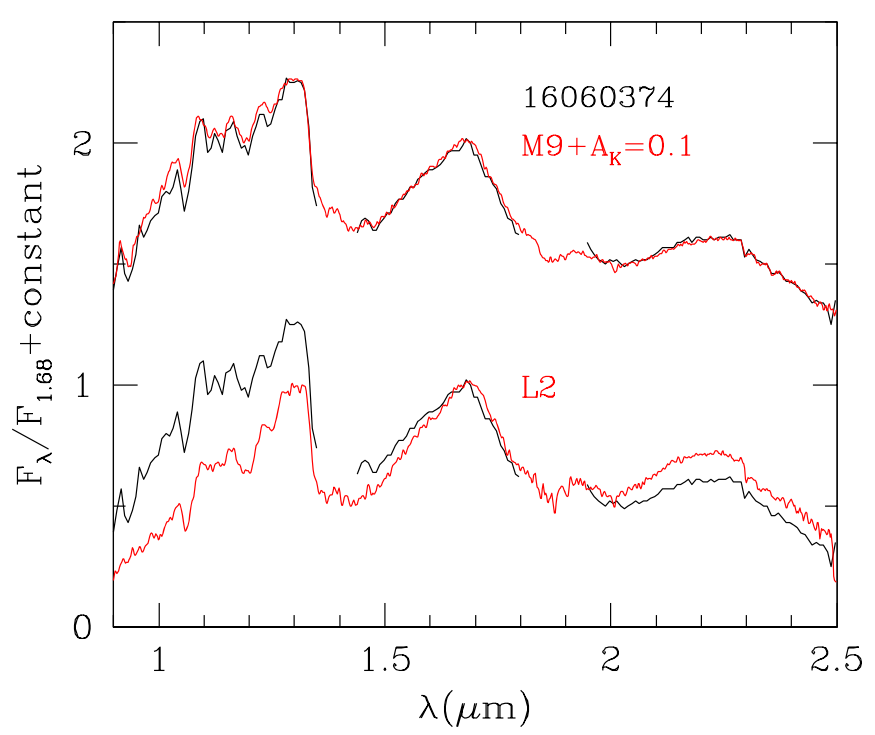

Figure 9. Example of a new reduction of an IR spectrum from Lodieu et al. (2008) for a late-type member of Upper Sco. It has been binned to a resolution of $R=150$. For comparison, we have included the young standard spectra from Luhman et al. (2017) for our adopted type of M9 and the type of L2 from Lodieu et al. (2008). The data used to create this figure are available.

We estimate spectral types of L0-L2 and M9.5-L2, respectively, whereas Peña Ramírez et al. (2016) and Lodieu et al. (2018) measured L6 for each of them. In Figure 10, we compare each of the two spectra to the standards from Luhman et al. (2017) that bracket our classifications. Among the L types, Luhman et al. (2017) adopted young standard spectra for L0, L2, L4, and L7. We have combined L4 and L7 with the appropriate weights to produce L6, and the resulting spectrum has been included in Figure 10. In our adopted classification system, UGCS J155150.21-213457.2 and UGCS J160413.03 -224103.2 are too blue to have types of L6. A similar result was found for 1RXS J160929.1-210524 B in Figure 18 from Luhman et al. (2017). It was previously classified as L4, but it is much bluer than our adopted L4 standard. Instead, Luhman et al. (2017) found a good match to M9.5 with $A_{V}=1.2$.

Most of our new classifications of previous IR spectra are earlier than the originally reported types. One reason for this difference is that we have accounted for the possibility of reddening from interstellar dust. For instance, a reddened early $\mathrm{L}$ dwarf and an unreddened late $\mathrm{L}$ dwarf can be indistinguishable in low-resolution spectra (Luhman et al. 2017). The choice of classification standards also contributes to the differences between our spectral types and values from other studies. In particular, the IR classifications from Lodieu et al. (2018) were largely based on comparison to normal (i.e., not young) field L dwarfs and the Upper Sco members 1RXS J160929.1 -210524 B and UGCS J155150.21-213457.2, for which they adopted the original types of L4 and L6. However, the latter two objects have earlier types when classified with our adopted standards, as we have discussed. In addition, the most prominent near-IR spectral features of late-type objects $\left(\mathrm{H}_{2} \mathrm{O}\right.$ bands and spectral slope) vary with surface gravity in such a way that using older field dwarfs as the standards for classifying young objects produces later types than if optically classified young standards are used (Luhman 2012, references therein). Indeed, Lodieu et al. (2018) found that their IR classifications (which they adopted) were systematically later than the optical types that they derived when both IR and optical spectra were available. We prefer a classification scheme that produces the same spectral types, on average, regardless of the wavelength range considered so that types 


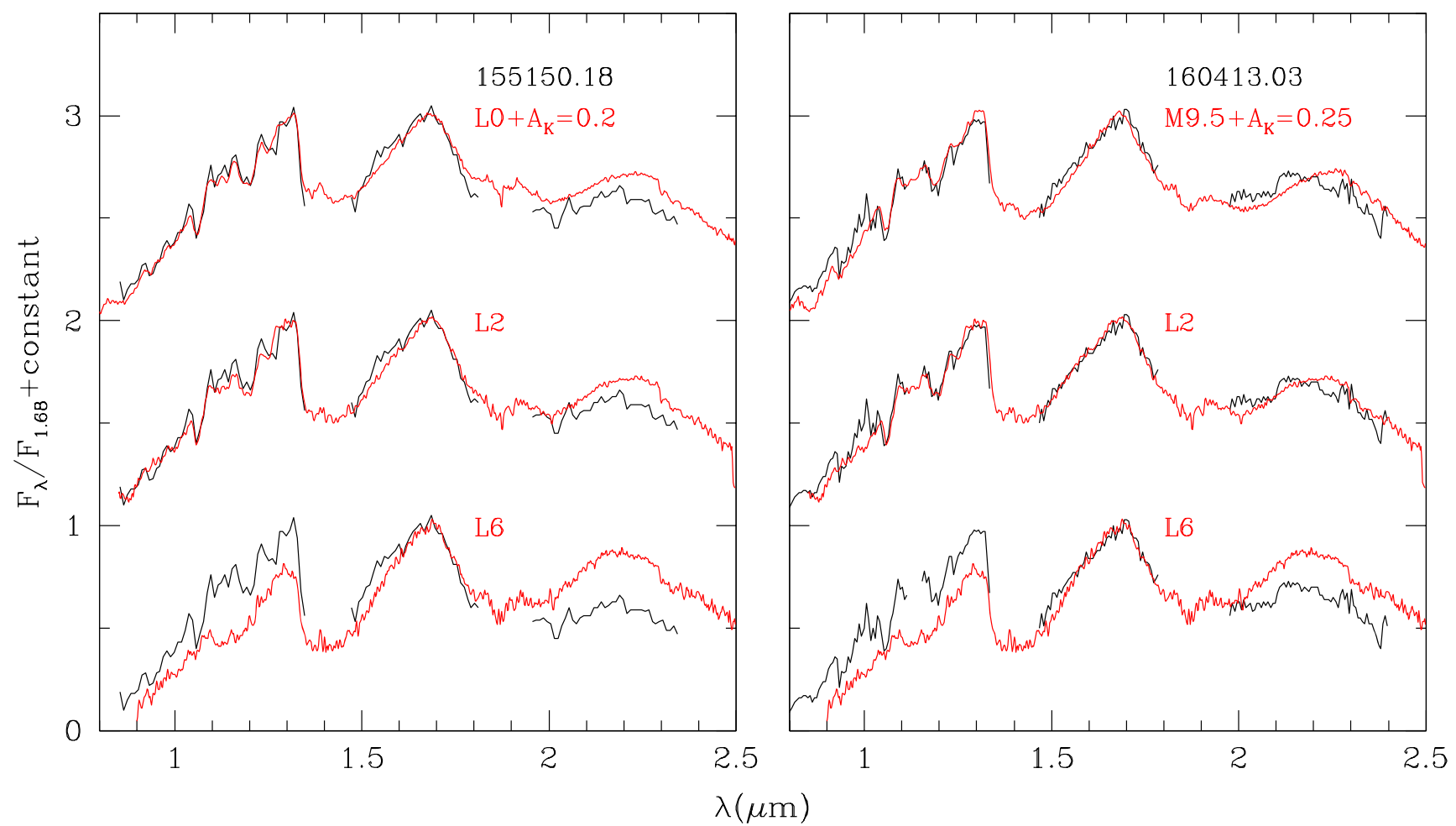

Figure 10. Examples of IR spectra of late-type members of Upper Sco from previous studies (Peña Ramírez et al. 2016; Lodieu et al. 2018) for which we have measured new spectral types. The data have been binned to a resolution of $R=150$. The spectrum of each object is compared to two young standards from Luhman et al. (2017) that bracket our derived spectral type (top and middle) and the young standard from Luhman et al. (2017) for the previously reported spectral type (bottom).

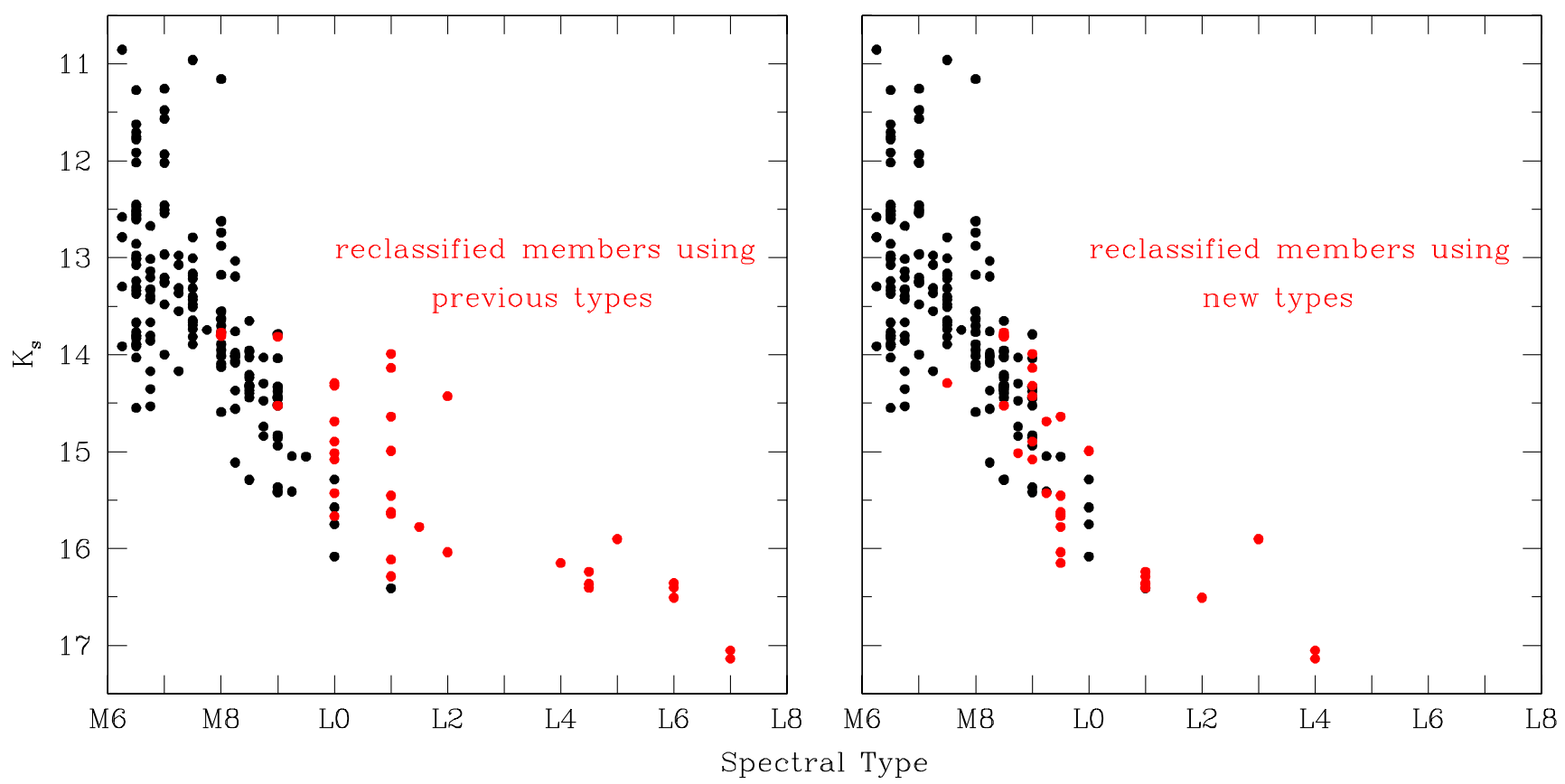

Figure 11. $K_{s}$ vs. spectral type for known late-type members of Upper Sco. We have measured new spectral types using IR spectra from previous studies for the objects with red symbols. That sample is plotted with the previous types (left) and our new types (right).

from different wavelengths can be utilized together in a meaningful way.

In Figure 11, we show two diagrams of $K_{s}$ versus spectral type for the known members of Upper Sco that are later than M6. For the objects that we have reclassified in this section, we have used the previous types in the left diagram and our new types in the right diagram. We have used the adopted types in Table 1 for all other members in both diagrams. The sequence of members is tighter and better defined when our new classifications are employed. 


\section{Future Prospects for Upper Sco Census}

The census of Upper Sco compiled by Luhman \& Mamajek (2012) contained 630 previously known members (excluding stars that we now reject). Subsequent spectroscopic surveys have identified hundreds of additional young stars that are likely to be members (Dawson et al. 2014; Rizzuto et al. 2015; Pecaut \& Mamajek 2016; Peña Ramírez et al. 2016; Lodieu et al. 2018, this work). Our latest sample of adopted members now contains 1631 objects. Based on the large number of additional candidates that we have identified $(>1000)$, substantial incompleteness likely remains in the current census of the association. The second data release from Gaia should provide highly precise measurements of proper motions and parallaxes down to substellar masses in Upper Sco, which will greatly facilitate the identification of the undiscovered members. The new Gaia data will also be valuable for identifying nonmembers that are present in the current census.

A relatively complete census of Upper Sco, particularly in conjunction with the astrometry from Gaia, will make it possible to address the following questions more definitively than has been possible previously: What is the shape of the IMF in Upper Sco and how does it compare to mass functions in other young nearby populations (Lodieu 2013)? How does the disk fraction vary with stellar mass and location (Luhman \& Mamajek 2012)? How does stellar age vary with stellar mass, disk class, and location (Donaldson et al. 2017)? Is a significant age spread present (Fang et al. 2017)? A full census of Upper Sco will also offer large, statistically significant samples of targets for measurements of stellar multiplicity and planet occurrence rates.

K.L. acknowledges support from NSF grant AST-1208239 and NASA grant 80NSSC18K0444. E.M. acknowledges support from the NASA NExSS program. Part of this research was carried out at the Jet Propulsion Laboratory (JPL), California Institute of Technology (Caltech), under a contract with NASA. The IRTF is operated by the University of Hawaii under contract NNH14CK55B with NASA. The data at CTIO and SOAR were obtained through programs 2011A-0415, 2013A-0224, 2014A-0180, 2015A-0192, and 2017A-0161 at the National Optical Astronomy Observatory (NOAO). CTIO and NOAO are operated by the Association of Universities for Research in Astronomy under a cooperative agreement with the NSF. The Gemini data were obtained through programs GS2009A-C-7 (NOAO 2009A-0083), GN-2017A-FT-12, and GN-2017A-FT-17. Gemini Observatory is operated by AURA under a cooperative agreement with the NSF on behalf of the Gemini partnership: the NSF (United States), the NRC (Canada), CONICYT (Chile), the ARC (Australia), Ministério da Ciência, Tecnologia e Inovação (Brazil) and Ministerio de Ciencia, Tecnología e Innovación Productiva (Argentina). WISE is a joint project of the University of California, Los Angeles, and the JPL/Caltech, funded by NASA. 2MASS is a joint project of the University of Massachusetts and IPAC at Caltech, funded by NASA and the NSF. This work used data from the NASA/IPAC Infrared Science Archive, operated by JPL under contract with NASA, and the SIMBAD database, operated at CDS, Strasbourg, France. The DENIS project was partly funded by the SCIENCE and the HCM plans of the European Commission under grants CT920791 and CT940627. It was supported by INSU, MEN and CNRS in France, by the State of Baden-Württemberg in Germany, by DGICYT in
Spain, by CNR in Italy, by FFwFBWF in Austria, by FAPESP in Brazil, by OTKA grants F-4239 and F-013990 in Hungary, and by the ESO C\&EE grant A-04-046. Jean Claude Renault from IAP was the Project manager. Observations were carried out thanks to the contribution of numerous students and young scientists from all involved institutes, under the supervision of P. Fouqué. The Pan-STARRS1 Surveys (PS1) and the PS1 public science archive have been made possible through contributions by the Institute for Astronomy, the University of Hawaii, the Pan-STARRS Project Office, the Max-Planck Society and its participating institutes, the Max Planck Institute for Astronomy, Heidelberg and the Max Planck Institute for Extraterrestrial Physics, Garching, The Johns Hopkins University, Durham University, the University of Edinburgh, the Queen's University Belfast, the Harvard-Smithsonian Center for Astrophysics, the Las Cumbres Observatory Global Telescope Network Incorporated, the National Central University of Taiwan, the Space Telescope Science Institute, the National Aeronautics and Space Administration under Grant NNX08AR22G issued through the Planetary Science Division of the NASA Science Mission Directorate, the NSF Grant AST-1238877, the University of Maryland, Eotvos Lorand University (ELTE), the Los Alamos National Laboratory, and the Gordon and Betty Moore Foundation. This work has made use of data from the European Space Agency (ESA) mission Gaia (https://www.cosmos.esa.int/gaia), processed by the Gaia Data Processing and Analysis Consortium (DPAC, https:// www.cosmos.esa.int/web/gaia/dpac/consortium). Funding for the DPAC has been provided by national institutions, in particular the institutions participating in the Gaia Multilateral Agreement. The Center for Exoplanets and Habitable Worlds is supported by the Pennsylvania State University, the Eberly College of Science, and the Pennsylvania Space Grant Consortium.

\section{ORCID iDs}

K. L. Luhman (1) https://orcid.org/0000-0003-2822-2951

K. A. Herrmann (1) https://orcid.org/0000-0003-0748-2316

E. E. Mamajek (i) https://orcid.org/0000-0003-2008-1488

T. L. Esplin (1) https://orcid.org/0000-0001-9223-9091

M. J. Pecaut (1) https://orcid.org/0000-0002-7859-1504

\section{References}

Aller, K. M., Kraus, A. L., Liu, M. C., et al. 2013, ApJ, 773, 63

Allers, K. N., Kessler-Silacci, J. E., Cieza, L. A., \& Jaffe, D. T. 2006, ApJ, 644,364

Allers, K. N., \& Liu, M. C. 2013, ApJ, 772, 79

Alonso, R., Deeg, H. J., Hoyer, S., et al. 2015, A\&A, 584, L8

Altmann, M., Roeser, S., Demleitner, M., Bastian, U., \& Schilbach, E. 2017, A\&A, 600, L4

Ansdell, M., Gaidos, E., Rappaport, S. A., et al. 2016, ApJ, 816, 69

Ansdell, M., Oelkers, R. J., Rodriguez, J. E., et al. 2018, MNRAS, 473, 1231

Ardila, D., Martín, E., \& Basri, G. 2000, AJ, 120, 479

Barenfeld, S. A., Carpenter, J. M., Ricci, L., \& Isella, A. 2016, ApJ, 827, 142

Barenfeld, S. A., Carpenter, J. M., Sargent, A. I., Isella, A., \& Ricci, L. 2017, ApJ, 851, 85

Béjar, V. J. S., Zapatero Osorio, M. R., Pérez-Garrido, A., et al. 2008, ApJL, $673, \mathrm{~L} 185$

Best, W. M. J., Liu, M. C., Magnier, E. A., et al. 2017, ApJ, 837, 95

Biller, B., Allers, K., Liu, M., Close, L. M., \& Dupey, T. 2011, ApJ, 730, 39 Bonnefoy, M., Chauvin, G., Lagrange, A.-M., et al. 2014, A\&A, 562, A127

Bouvier, J., \& Appenzeller, I. 1992, A\&AS, 92, 481

Bouy, H., \& Martín, E. L. 2009, A\&A, 504, 981

Bowler, B. P., Kraus, A. L., Bryan, M. L., et al. 2017, AJ, 154, 165

Bowler, B. P., Liu, M. C., Kraus, A. L., \& Mann, A. W. 2014, ApJ, 784, 65 
Bowler, B. P., Liu, M. C., Kraus, A. L., Mann, A. W., \& Ireland, M. J. 2011, ApJ, 743, 148

Brandner, W., \& Zinnecker, H. 1997, A\&A, 321, 220

Carpenter, J. M., Bouwman, J., Silverstone, M. D., et al. 2008, ApJS, 179, 423

Carpenter, J. M., Mamajek, E. E., Hillenbrand, L. A., \& Meyer, M. R. 2006, ApJL, 651, L49

Carpenter, J. M., Mamajek, E. E., Hillenbrand, L. A., \& Meyer, M. R. 2009, ApJ, 705, 1646

Carpenter, J. M., Ricci, L., \& Isella, A. 2014, ApJ, 787, 42

Chabrier, G., \& Baraffe, I. 1997, A\&A, 327, 1039

Chambers, K. C., Magnier, E. A., Metcalfe, N., et al. 2016, arXiv: 1612.05560

Chen, C. H., Jura, M., Gordon, K. D., \& Blaylock, M. 2005, ApJ, 623, 493

Chen, C. H., Mamajek, E. E., Bitner, M. A., et al. 2011, ApJ, 738, 122

Cieza, L., Padgett, D. L., Stapelfeldt, K. R., et al. 2007, ApJ, 667, 308

Cieza, L. A., Schreiber, M. R., Romero, G. A., et al. 2010, ApJ, 712, 925

Close, L. M., Zuckerman, B., Song, I., et al. 2007, ApJ, 660, 1492

Cody, A. M., \& Hillenbrand, L. A. 2018, AJ, in press

Cody, A. M., Hillenbrand, L. A., David, T. J., et al. 2017, ApJ, 836, 41

Cohen, M., \& Kuhi, L. V. 1979, ApJS, 41, 743

Cook, N. J., Scholz, A., \& Jayawardhana, R. 2017, AJ, 154, 256

Corbally, C. J. 1984, ApJS, 285, 195

Cowley, A., Cowley, C., Jaschek, M., \& Jaschek, C. 1969, AJ, 74, 375

Cruz, K. L., Kirkpatrick, J. D., \& Burgasser, A. J. 2009, AJ, 137, 3345

Cruz, K. L., Núñez, A., Burgasser, A. J., et al. 2018, AJ, 155, 34

Cruz, K. L., Reid, I. N., Kirkpatrick, J. D., et al. 2007, AJ, 133, 439

Cushing, M. C., Rayner, J. T., \& Vacca, W. D. 2005, ApJ, 623, 1115

Cushing, M. C., Vacca, W. D., \& Rayner, J. T. 2004, PASP, 116, 362

Dahm, S. E., \& Carpenter, J. M. 2009, AJ, 137, 4024

Danielski, C., Babusiaux, C., Ruiz-Dern, L., Sartoretti, P., \& Arenou, F. 2018, A\&A, 614, A19

D’Antona, F., \& Mazzitelli, I. 1997, MmSAI, 68, 807

David, T. J., Hillenbrand, L. A., Cody, A. M., Carpenter, J. M., \& Howard, A. W. 2016a, ApJ, 816, 21

David, T. J., Hillenbrand, L. A., Petigura, E. A., et al. 2016b, Natur, 534, 658

David, T. J., Petigura, E. A., Hillenbrand, L. A., et al. 2017, ApJ, 835, 168

Dawson, P., Scholz, A., \& Ray, T. P. 2011, MNRAS, 418, 1231

Dawson, P., Scholz, A., Ray, T. P., et al. 2013, MNRAS, 429, 903

Dawson, P., Scholz, A., Ray, T. P., et al. 2014, MNRAS, 442, 1586

de Bruijne, J. H. J. 2012, Ap\&SS, 341, 31

de Zeeuw, P. T., Hoogerwerf, R., de Bruijne, J. H. J., Brown, A. G. A., \& Blaauw, A. 1999, AJ, 117, 354

Dobashi, K., Uehara, H., Kandori, R., et al. 2005, PASJ, 57, 1

Donaldson, J. K., Weinberger, A. J., Gagné, J., Boss, A. P., \& Keiser, S. A. 2017, ApJ, 850, 11

Eisner, J. A., Hillenbrand, L. A., White, R. J., Akeson, R. L., \& Sargent, A. I. 2005, ApJ, 623, 952

Elias, J. H., Joyce, R. R., Liang, M., et al. 2006, Proc. SPIE, 6269, 62694C

Epchtein, N., Deul, E., Derriere, S., et al. 1999, A\&A, 349, 236

Erickson, K. L., Wilking, B. A., Meyer, M. R., Robinson, J. G., \& Stephenson, L. N. 2011, AJ, 142, 140

Esplin, T. L., \& Luhman, K. L. 2017, AJ, 154, 134

Esplin, T. L., Luhman, K. L., Miller, E. B., \& Mamajek, E. E. 2018, AJ, in press

Faherty, J. K., Riedel, A. R., Cruz, K. L., et al. 2016, ApJS, 225, 10

Fang, Q., Herczeg, G. J., \& Rizzuto, A. 2017, ApJ, 842, 123

Feiden, G. A. 2016, A\&A, 593, A99

Filippazzo, J. C., Giorla Godfrey, P., Cruz, K. L., et al. 2016, The BDNYC Database v1.0, Zenodo, doi:10.5281/zenodo.45169

Findeisen, K., \& Hillenbrand, L. 2010, AJ, 139, 1338

Flewelling, H. A., Magnier, E. A., Chambers, K. C., et al. 2016, arXiv:1612. 05243

Gaia Collaboration, Brown, A. G. A., Vallenari, A., et al. 2016a, A\&A, $595, \mathrm{~A} 2$

Gaia Collaboration, Prusti, T., de Bruijne, J. H. J., et al. 2016b, A\&A, 595, A1

Gizis, J. E. 2002, ApJ, 575, 484

Gray, R. O., Corbally, C., Garrison, R. F., et al. 2006, AJ, 132, 161

Hedges, C., Hodgkin, S., \& Kennedy, G. 2018, MNRAS, 476, 2968

Henry, T. J., Kirkpatrick, J. D., \& Simons, D. A. 1994, AJ, 108, 1437

Herbig, G. H., \& Kameswara Rao, N. 1972, ApJ, 174, 401

Herczeg, G. J., Cruz, K. L., \& Hillenbrand, L. A. 2009, ApJ, 696, 1589

Hinkley, S., Kraus, A. L., Ireland, M. J., et al. 2015, ApJL, 806, L9

Hoogerwerf, R. 2000, MNRAS, 313, 43

Hook, I., Jørgensen, I., Allington-Smith, J. R., et al. 2004, PASP, 116, 425

Houk, N. 1982, Michigan Catalogue of Two-dimensional Spectral Types for the HD Stars, Vol. 3 (Ann Arbor: Univ. Mich.)
Houk, N., \& Smith-Moore, M. 1988, Michigan Catalogue of Two-dimensional Spectral Types for the HD Stars, Vol. 4 (Ann Arbor: Univ. Mich.)

Indebetouw, R., Mathis, J. S., Babler, B. L., et al. 2005, ApJ, 619, 931

Ireland, M. J., Kraus, A., Martinache, F., Law, N., \& Hillenbrand, L. A. 2011, ApJ, 726, 113

Jang-Condell, H., Chen, C. H., Mittal, T., et al. 2015, ApJ, 808, 167

Jayawardhana, R., \& Ivanov, V. D. 2006, ApJL, 647, L167

Kaiser, N., Aussel, H., Burke, B. E., et al. 2002, Proc. SPIE, 4836, 154

Kaiser, N., Burgett, W., Chambers, K., et al. 2010, Proc. SPIE, 7733, 12

Keenan, P. C., \& McNeil, R. C. 1989, ApJS, 71, 245

Kellogg, K., Metchev, S., Geißler, K., et al. 2015, AJ, 150, 182

Kenyon, S. J., \& Hartmann, L. 1995, ApJS, 101, 117

Kirkpatrick, J. D., Barman, T. S., Burgasser, A. J., et al. 2006, ApJ, 639, 1120

Kirkpatrick, J. D., Henry, T. J., \& Irwin, M. J. 1997, AJ, 113, 1421

Kirkpatrick, J. D., Henry, T. J., \& McCarthy, D. W. 1991, ApJS, 77, 417

Kirkpatrick, J. D., Looper, D. L., Burgasser, A. J., et al. 2010, ApJS, 190, 100

Kraus, A. L., Cody, A. M., Covey, K. R., et al. 2015, ApJ, 807, 3

Kraus, A. L., \& Hillenbrand, L. A. 2007, ApJ, 664, 1167

Kraus, A. L., \& Hillenbrand, L. A. 2009, ApJ, 703, 1511

Kraus, A. L., Ireland, M. J., Martinache, F., \& Lloyd, J. P. 2008, ApJ, 679, 762

Kraus, A. L., White, R. J., \& Hillenbrand, L. A. 2005, ApJ, 633, 452

Kunkel, M. 1999, PhD thesis, Julius-Maximilians-Univ.

Lachapelle, F.-R., Lafrenière, D., Gagné, J., et al. 2015, ApJ, 802, 61

Lafrenière, D., Jayawardhana, R., Janson, M., et al. 2011, ApJ, 730, 42

Lafrenière, D., Jayawardhana, R., \& van Kerkwijk, M. H. 2008, ApJL, 689, L153

Lafrenière, D., Jayawardhana, R., \& van Kerkwijk, M. H. 2010, ApJ, 719, 497

Lafrenière, D., Jayawardhana, R., van Kerkwijk, M. H., Brandeker, A., \& Janson, M. 2014, ApJ, 785, 47

Lawrence, A., Warren, S. J., Almaini, O., et al. 2007, MNRAS, 379, 1599

Lodieu, N. 2013, MNRAS, 431, 3222

Lodieu, N., Alonso, R., González Hernández, J. I., et al. 2015, A\&A, 584, A128

Lodieu, N., Dobbie, P. D., Cross, N. J. G., et al. 2013a, MNRAS, 435, 2474

Lodieu, N., Dobbie, P. D., \& Hambly, N. C. 2011a, A\&A, 527, A24

Lodieu, N., Hambly, N. C., Dobbie, P. D., et al. 2011b, MNRAS, 418, 2604

Lodieu, N., Hambly, N. C., \& Jameson, R. F. 2006, MNRAS, 373, 95

Lodieu, N., Hambly, N. C., Jameson, R. F., et al. 2007, MNRAS, 374, 372

Lodieu, N., Hambly, N. C., Jameson, R. F., \& Hodgkin, S. T. 2008, MNRAS, 383,1385

Lodieu, N., Ivanov, V. D., \& Dobbie, P. D. 2013b, MNRAS, 430, 1784

Lodieu, N., Zapatero Osorio, M. R., Béjar, V. J. S., \& Peña Ramírez, K. 2018 , MNRAS, 473, 2020

Luhman, K. L. 1999, ApJ, 525, 466

Luhman, K. L. 2005, ApJL, 633, L41

Luhman, K. L. 2012, ARA\&A, 50, 65

Luhman, K. L., Allen, P. R., Espaillat, C., Hartmann, L., \& Calvet, N. 2010, ApJS, 186, 111

Luhman, K. L., Allers, K. N., Jaffe, D. T., et al. 2007, ApJ, 659, 1629

Luhman, K. L., Briceño, C., Stauffer, J. R., et al. 2003, ApJ, 590, 348

Luhman, K. L., Liebert, J., \& Rieke, G. H. 1997, ApJL, 489, L165

Luhman, K. L., \& Mamajek, E. E. 2012, ApJ, 758, 31

Luhman, K. L., Mamajek, E. E., Shukla, S. J., \& Loutrel, N. P. 2017, AJ, 153,46

Luhman, K. L., Rieke, G. H., Lada, C. J., \& Lada, E. A. 1998, ApJ, 508, 347

Lutz, T. E., \& Lutz, J. H. 1977, AJ, 82, 431

Mann, A. W., Newton, E. R., Rizzuto, A. C., et al. 2016, AJ, 152, 61

Martín, E. L. 1998, AJ, 115, 351

Martín, E. L., Delfosse, X., \& Guieu, S. 2004, AJ, 127, 449

Martín, E. L., Montmerle, T., Gregorio-Hetem, J., \& Casanova, S. 1998, MNRAS, 300, 733

Martín, E. L., Phan-Bao, N., Bessell, M., et al. 2010, A\&A, 517, A53

Martini, P., Stoll, R., Derwent, M. A., et al. 2011, PASP, 123, 187

Mathews, G. S., Pinte, C., Duchêne, G., Williams, J. P., \& Ménard, F. 2013, A\&A, 558, A66

Mathews, G. S., Williams, J. P., Ménard, F., et al. 2012, ApJ, 745, 23

McClure, M. K., Furlan, E., Manoj, P., et al. 2010, ApJS, 188, 75

McMahon, R. G., Banerji, M., Gonzalez, E., et al. 2013, Msngr, 154, 35

Mellon, S. N., Mamajek, E. E., Oberst, T. E., \& Pecaut, M. J. 2017, ApJ, 844,16

Merín, B., Brown, J. M., Oliveira, I., et al. 2010, ApJ, 718, 1200

Mora, A., Merin, B., Solano, E., et al. 2001, A\&A, 378, 116

Muzerolle, J., Hillenbrand, L., Calvet, N., Briceño, C., \& Hartmann, L. 2003, ApJ, 592, 266

Neuhäuser, R., Torres, G., Sterzik, M. F., \& Randich, S. 1997, A\&A, 325, 647

Nordström, B., Mayor, M., Andersen, J., et al. 2004, A\&A, 418, 989 
Padgett, D. L., Cieza, L., Stapelfeldt, K. R., et al. 2006, ApJ, 645, 1283

Pecaut, M. J., \& Mamajek, E. E. 2016, MNRAS, 461, 794

Pecaut, M. J., Mamajek, E. E., \& Bubar, E. J. 2012, ApJ, 746, 154

Peña Ramírez, K., Béjar, V. J. S., \& Zapatero Osorio, M. R. 2016, A\&A, 586, A157

Perryman, M. A. C., de Boer, K. S., Gilmore, G., et al. 2001, A\&A, 369, 339 Prato, L. 2007, ApJ, 657, 338

Prato, L., Greene, T. P., \& Simon, M. 2003, ApJ, 584, 853

Preibisch, T., Brown, A. G. A., Bridges, T., Guenther, E., \& Zinnecker, H. 2002, AJ, 124, 404

Preibisch, T., Guenther, E., Zinnecker, H., et al. 1998, A\&A, 333, 619

Preibisch, T., Guenther, E., \& Zinnecker, H. 2001, AJ, 121, 1040

Preibisch, T., \& Mamajek, E. 2008, in Handbook of Star-forming Regions,

Vol. 2, The Southern Sky, ed. B. Reipurth (San Francisco, CA: ASP), 235

Rayner, J. T., Cushing, M. C., \& Vacca, W. D. 2009, ApJS, 185, 289

Rayner, J. T., Toomey, D. W., Onaka, P. M., et al. 2003, PASP, 115, 362

Rebull, L. M., Stauffer, J. R., Cody, A. M., et al. 2018, AJ, 155, 196

Reid, I. N., Cruz, K. L., Kirkpatrick, J. D., et al. 2008, AJ, 136, 1290

Riaz, B., Gizis, J. E., \& Harvin, J. 2006, AJ, 132, 866

Riaz, B., Lodieu, N., \& Gizis, J. E. 2009, ApJ, 705, 1173

Riaz, B., Lodieu, N., Goodwin, S., Stamatellos, D., \& Thompson, M. 2012, MNRAS, 420, 2497

Rieke, G. H., Su, K. Y. L., Stansberry, J. A., et al. 2005, ApJ, 620, 1010 Ripepi, V., Balona, L., Catanzaro, G., et al. 2015, MNRAS, 454, 2606

Rizzuto, A. C., Ireland, M. J., \& Kraus, A. L. 2015, MNRAS, 448, 2737

Rizzuto, A. C., Ireland, M. J., \& Robertson, J. G. 2011, MNRAS, 416, 3108

Rizzuto, A. C., Ireland, M. J., \& Zucker, D. B. 2012, MNRAS, 421, L97

Rizzuto, A. C., Mann, A. W., Vanderburg, A., Kraus, A. L., \& Covey, K. R. 2017, AJ, 154, 224

Romero, G. A., Schreiber, M. R., Cieza, L. A., et al. 2012, ApJ, 749, 79
Ruiz, M. T., Maza, J., Gonzalez, L. E., \& Wischnjewsky, M. 1987, AJ, 94, 1299

Scaringi, S., Manara, C. F., Barenfeld, S. A., et al. 2016, MNRAS, 463, 2265

Schlafly, E. F., Meisner, A. M., Stutz, A. M., et al. 2016, ApJ, 821, 78

Schneider, A. C., Windsor, J., Cushing, M. C., Kirkpatrick, J. D., \& Wright, E. L. 2016, ApJL, 822, L1

Scholz, A., Jayawardhana, R., Wood, K., et al. 2007, ApJ, 660, 1517

Scholz, A., Kostov, V., Jayawardhana, R., \& Mužić, K. 2015, ApJL, 809, L29

Shkolnik, E., Liu, M. C., \& Reid, I. N. 2009, ApJ, 699, 649

Siess, L., Dufour, E., \& Forestini, M. 2000, A\&A, 358, 593

Skrutskie, M., Cutri, R. M., Stiening, R., et al. 2006, AJ, 131, 1163

Slesnick, C. L., Carpenter, J. M., \& Hillenbrand, L. A. 2006, AJ, 131, 3016

Slesnick, C. L., Hillenbrand, L. A., \& Carpenter, J. M. , 2008, ApJ, 688, 377

Stauffer, J., Collier-Cameron, A., Jardine, M., et al. 2017, AJ, 153, 152

Stauffer, J., Rebull, L., David, T. J., et al. 2018, AJ, 155, 63

Tian, H.-J., Gupta, P., Sesar, B., et al. 2017, ApJS, 232, 4

Tonry, J. L., Stubbs, C. W., Lykke, K. R., et al. 2012, ApJ, 750, 99

Torres, C. A. O., Quast, G. R., Da Silva, L., et al. 2006, A\&A, 460, 695

Uyama, T., Hashimoto, J., Kuzuhara, M., et al. 2017, AJ, 153, 106

Vacca, W. D., Cushing, M. C., \& Rayner, J. T. 2003, PASP, 115, 389

Vieira, S. L. A., Corradi, W. J. B., Alencar, S. H. P., et al. 2003, AJ, 126, 2971

Walter, F. M., Vrba, F. J., Mathieu, R. D., Brown, A., \& Myers, P. C. 1994, AJ, 107, 692

Wilking, B. A., Meyer, M. R., Robinson, J. G., \& Greene, T. P. 2005, AJ, 130,1733

Wilkinson, S., Merín, B., \& Riviere-Marichalar, P. 2018, A\&A, in press

Wright, E. L., Eisenhardt, P. R., Mainzer, A. K., et al. 2010, AJ, 140, 1868

Wright, N. J., \& Mamajek, E. E. 2018, MNRAS, 476, 381

Xue, M., Jiang, B. W., Liu, J., et al. 2016, ApJS, 224, 23

Zacharias, N., Finch, C., \& Frouard, J. 2017, AJ, 153, 166 